TRANSACTIONS OF THE

AMERICAN MATHEMATICAL SOCIETY

Volume 349, Number 9, September 1997, Pages 3737-3771

S $0002-9947(97) 01839-4$

\title{
THE BRAUER GROUP OF YETTER-DRINFEL'D MODULE ALGEBRAS
}

\author{
S. CAENEPEEL, F. VAN OYSTAEYEN, AND Y. H. ZHANG
}

\begin{abstract}
Let $H$ be a Hopf algebra with bijective antipode. In a previous paper, we introduced $H$-Azumaya Yetter-Drinfel'd module algebras, and the Brauer group $\mathrm{BQ}(k, H)$ classifying them. We continue our study of $\mathrm{BQ}(k, H)$, and we generalize some properties that were previously known for the BrauerLong group. We also investigate separability properties for $H$-Azumaya algebras, and this leads to the notion of strongly separable $H$-Azumaya algebra, and to a new subgroup of the Brauer group $\mathrm{BQ}(k, H)$.
\end{abstract}

\section{INTRODUCTION}

Let $k$ be a commutative ring. Wall [26] introduced a Brauer group of $\mathbb{Z} / 2 \mathbb{Z}$ graded algebras. During the early seventies, some generalizations to gradings by other groups have been proposed, cf. e.g. [9]. In [14], Long introduced a Brauer group of $H$-dimodule algebras (that is, algebras with an $H$-action and an $H$-coaction), where $H$ is a finitely generated, projective, commutative and cocommutative Hopf algebra. Long's Brauer group, today usually referred to as the Brauer-Long group, turned out to be the appropriate generalization of the BrauerWall group, since it contains all previously proposed generalizations as subgroups.

Several authors have investigated the properties of the Brauer-Long group, cf. e.g. $[1,2,5,6,7,10,19,20]$ (this list is not exhaustive). In all computations, the fact that the basic Hopf algebra is finitely generated, projective, commutative and cocommutative seems to be essential. This is a severe restriction, since, apart from finite abelian group rings, examples of such Hopf algebras are scarce. In a previous paper [8], we have been able to generalize Long's construction to arbitrary Hopf algebras - the only remaining condition is that the Hopf algebra $H$ needs to have a bijective antipode. The underlying philosophy is that we replace Long's dimodules and dimodule algebras by Yetter-Drinfel'd modules (also called quantum YangBaxter modules or crossed modules) and Yetter-Drinfel'd module algebras. The Brauer group $\mathrm{BQ}(k, H)$ then consists of Brauer equivalence classes of $H$-Azumaya algebras ; if $H$ is finitely generated, projective, commutative and cocommutative, then $\mathrm{BQ}(k, H)$ is anti-isomorphic to the Brauer-Long group $\mathrm{BD}(k, H)$. The fact that we have an anti-isomorphism rather than an isomorphism comes from the

Received by the editors August 24, 1994 and, in revised form, March 19, 1996.

1991 Mathematics Subject Classification. Primary 16A16, 16A24.

Key words and phrases. Brauer group, Azumaya algebra, Hopf algebra, Yetter-Drinfel'd module, separable algebra.

The third author wishes to thank the Free University of Brussels for its financial support during the time when this paper was written. 
fact that we took the braided product instead of the smash product to define the multiplication on $\mathrm{BQ}(k, H)$.

In this paper, we continue our study of $\mathrm{BQ}(k, H)$. Some of our results are generalizations of results that were previously obtained for the Brauer-Long group. In Section 1, we fix notations, and recall some definitions from [8]. We also introduce a generalization of the Brauer group of $H$-comodule algebras (cf. [1, 2]). If $(H, R)$ is a quasitriangular Hopf algebra, then we introduce the $\operatorname{Brauer}$ group $\operatorname{BM}(k, H, \mathcal{R})$. A similar generalization of the Brauer group of $H$-module algebras is possible for a coquasitriangular Hopf algebra. The Brauer group $\mathrm{BC}(k, H, \mathcal{R})$ thus obtained is also a generalization of the Brauer groups introduced in [9] and [19]. If $H$ is finite, then it is known that the Drinfel'd double is quasitriangular, and in this case $\mathrm{BQ}(k, H)=\mathrm{BM}(k, D(H), R)$, and this generalizes an observation made by Childs, cf. $[9,10]$.

In Section 2, we introduce the notion of Hopf Yetter-Drinfel'd $(A, H)$-module, and we discuss a Morita theory for these modules. Roughly stated, a YetterDrinfel'd module algebra $A$ is $H$-Azumaya if and only if the categories of YetterDrinfel'd $H$-modules and Hopf Yetter-Drinfel'd $(A, H)$-module algebras are equivalent, cf. Proposition 2.6. This generalizes some results of [1].

We then investigate to what extent an $H$-Azumaya algebra may be defined as a kind of central separable algebra. This problem was studied in the classical case in [1], where the notions of $H$-central and $H$-separable algebra were introduced. Using the Morita theory, it is not difficult to show that an $H$-Azumaya algebra is $H$-central and $H$-separable. In general, the converse does not hold. We will follow a different approach, which is, as far as we could find out, also new in the classical case of a faithfully projective, commutative, cocommutative Hopf algebra. We introduce the notion of strongly $H$-separable algebra, which differs from an ordinary $H$-separable algebra in the sense that the $H$-separability idempotent has to be in the intersection of the invariants and coinvariants of the $H$-enveloping algebra $A^{\# e}$. A strongly $H$ separable $H$-central algebra is then called strongly $H$-Azumaya, and we can show that a strongly $H$-Azumaya algebra is automatically $H$-Azumaya, cf. Theorem 2.23. The converse is not true, however, and the subset of $\mathrm{BQ}(k, H)$ consisting of classes represented by a strongly $H$-Azumaya algebra forms a subgroup denoted by $\operatorname{BQS}(k, H)$, cf. Theorem 2.26. We can characterize strong $H$-Azumaya algebras as $H$-Azumaya algebras having a trace map, cf. Proposition 2.24. If $H$ is semisimple and cosemisimple, then we can show that $\mathrm{BQS}(k, H)=\mathrm{BQ}(k, H)$.

It was established long ago that there is no splitting theorem for the BrauerLong group, nor even for the Brauer-Wall group. In particular, the Brauer-Long group of an algebraically closed field may be nontrivial, for example $\mathrm{BD}(\mathbb{C}, \mathbb{Z} / 2 \mathbb{Z})=$ $\mathbb{Z} / 2 \mathbb{Z}$. Denoting by $\mathrm{BQ}^{s}(k, H)$ the subgroup of $\mathrm{BQ}(k, H)$ of classes of algebras split by a faithfully flat extension of $k$, it makes sense to try to describe the cokernel of the inclusion $\mathrm{BQ}^{s}(k, H) \longrightarrow \mathrm{BQ}(k, H)$. In the case of a faithfully projective, commutative and cocommutative Hopf algebra, and following methods based on the Skolem-Noether theorem, originally considered in a special case by Orzech ([20]), it was shown in [6] that we have an exact sequence

$$
1 \longrightarrow \mathrm{BD}^{s}(k, H) \longrightarrow \mathrm{BD}(k, H) \longrightarrow \mathrm{O}(k, H)
$$

where $\mathrm{O}(k, H)$ is a well-determined kind of orthogonal subgroup of the automorphism group $\operatorname{Aut}_{\text {Hopf }}\left(H \otimes H^{*}\right)$. In Section 2, we try to extend this result. We first 
give a Yetter-Drinfel'd version of the Rosenberg-Zelinsky sequence and the SkolemNoether theorem (cf. Propositions 3.5 and 3.6). We may then prove a weak version of (1), namely, for a faithfully projective Hopf algebra $H$, we have a complex

$$
1 \longrightarrow \mathrm{BQ}^{s}(k, H) \longrightarrow \mathrm{BQ}(k, H) \longrightarrow \mathrm{O}(E(H)),
$$

where $\mathrm{O}(E(H))$ is a well-defined subgroup of $G(H) \times \operatorname{Alg}(H, k)$, cf. [20]. We expect that the quotient $\mathrm{BQ}(k, H) / \mathrm{BQ}^{s}(k, H)$ is a subgroup of the Hopf automorphism group of the Drinfel'd double $\mathrm{D}(H)$, but we have no idea how to prove this. The methods used in [6] are strongly based on the fact that the Hopf algebra is commutative and cocommutative.

In the final section, we show that there is an anti-homomorphism

$$
\pi: \operatorname{Aut}_{\text {Hopf }}(H) \longrightarrow \mathrm{BQ}(k, H)
$$

and this (partly) generalizes a result of Deegan [11], who showed that, for a finite abelian group $G, \operatorname{Aut}(G)$ is a subgroup of $\operatorname{BD}(k, k G)$. Moreover, using the map $\pi$ we may characterize the action of $\operatorname{Aut}_{\text {Hopf }}(H)$ on $\mathrm{BQ}(k, H)$, cf. Theorem 4.11.

An important property of the category of Yetter-Drinfel'd modules is that they form a braided category. The second and the third author observed that the construction of the Brauer group of Yetter-Drinfel'd module algebras can be generalized to arbitrary braided monoidal categories. This unifies almost all variations of the Brauer group that have been presented in the literature so far. In particular, if the braiding on the category is trivial, then one obtains the Brauer group of a symmetric monoidal category as introduced by Pareigis in [21]. For details, we refer to the forthcoming [25].

\section{Preliminaries}

In this section we review some notions about Hopf algebras, Yetter-Drinfel'd module algebras and the Brauer group of a Hopf algebra. For all unexplained terminology we refer to $[8,24]$. Throughout this paper $H$ is a Hopf algebra with bijective antipode $S$. Then $H^{o p}$ and $H^{c o p}$ are also Hopf algebras with antipode $S^{-1}$

1.1. Yetter-Drinfel'd $H$-module algebras. A Yetter-Drinfel' $d H$-module (cf. [8, $15,23,27]$ ), sometimes also called an $H$-crossed module or a quantum Yang-Baxter $H$-module, is a $k$-module $M$ which is at once a left $H$-module and a right $H$ comodule satisfying the compatibility relation

$$
\chi(h \cdot a)=\sum h_{(2)} \cdot a_{(0)} \otimes h_{(3)} a_{(1)} S^{-1}\left(h_{(1)}\right)
$$

A Yetter-Drinfel' $d H$-module algebra $A$ is a $k$-algebra which is a left $H$-module algebra and a right $H^{o p}$-comodule algebra satisfying the condition (3). As pointed out in [8], $\left(H^{o p}, \Delta, \mathrm{ad}^{\prime}\right)$ and $(H, \chi$, ad $)$ are regular Yetter-Drinfel'd $H$-module algebras with $H$-structures defined as follows:

$$
\begin{aligned}
h \operatorname{ad}^{\prime} x & =\sum h_{(2)} x S^{-1}\left(h_{(1)}\right) \\
\Delta(x) & =\sum x_{(1)} \otimes x_{(2)} \\
h \operatorname{ad} x & =\sum h_{(1)} x S\left(h_{(2)}\right) \\
\chi(x) & =\sum x_{(2)} \otimes S^{-1}\left(x_{(1)}\right)
\end{aligned}
$$


Let $A$ be a Yetter-Drinfel'd $H$-module algebra. The $H$-opposite algebra $\bar{A}$ of $A$ is the Yetter-Drinfel'd $H$-module algebra defined as follows: $\bar{A}$ equals $A$ as a Yetter-Drinfel'd $H$-module, with multiplication given by the formula

$$
\bar{a} \circ \bar{b}=\sum \overline{b_{(0)}\left(b_{(1)} \cdot a\right)}
$$

for all $\bar{a}, \bar{b} \in \bar{A}$. If the antipode of $H$ is of order 2, then $\overline{\bar{A}}$ is isomorphic to $A$ as a Yetter-Drinfel'd $H$-module algebra.

Let $M$ be a Yetter-Drinfel'd $H$-module which is faithfully projective as a $k$ module, that is, $M$ is finitely generated, projective and faithful as a $k$-module. The endomorphism algebra $\operatorname{End}_{k}(M)$ is a Yetter-Drinfel'd $H$-module algebra with the $H$-structures induced by those of $M$, i.e, for $h \in H, f \in \operatorname{End}_{k}(M)$ and $m \in M$,

$$
(h \cdot f)(m)=\sum h_{(1)} \cdot f\left(S\left(h_{(2)}\right) \cdot m\right)
$$

and

$$
\chi(f)(m)=\sum f\left(m_{(0)}\right)_{(0)} \otimes S^{-1}\left(m_{(1)}\right) f\left(m_{(0)}\right)_{(1)}
$$

Recall from $[8,4.2]$ that the $H$-opposite of $\operatorname{End}_{k}(M)$ is isomorphic to $\operatorname{End}_{k}(M)^{o p}$ as an $R$-algebra, with $H$-module structures given by

$$
(h \cdot f)(m)=\sum h_{(2)} \cdot f\left(S^{-1}\left(h_{(1)}\right) \cdot m\right)
$$

and

$$
\chi(f)(m)=\sum f\left(m_{(0)}\right)_{(0)} \otimes f\left(m_{(0)}\right)_{(1)} S\left(m_{(0)}\right)
$$

for $m \in M, h \in H$ and $f \in \operatorname{End}_{k}(M)$.

1.2. Quasitriangular Hopf algebras. A quasitriangular Hopf algebra is a pair $(H, R)$, where $H$ is a Hopf algebra with bijective antipode and $R=\sum R^{(1)} \otimes R^{(2)} \in$ $H \otimes H$ satisfies the following axioms $(r=R)$ :

$$
\begin{array}{ll}
\text { (QT1). } & \sum \Delta\left(R^{(1)}\right) \otimes R^{(2)}=\sum R^{(1)} \otimes r^{(1)} \otimes R^{(2)} r^{(2)} \\
\text { (QT2). } & \sum \varepsilon\left(R^{(1)}\right) R^{(2)}=1 \\
\text { (QT3). } & \sum R^{(1)} \otimes \Delta\left(R^{(2)}\right)=\sum R^{(1)} r^{(1)} \otimes r^{(2)} \otimes R^{(2)} \\
\text { (QT4). } & \sum R^{(1)} \varepsilon\left(R^{(2)}\right)=1 \\
\text { (QT5). } & \Delta^{c o p}(h) R=R \Delta(h)
\end{array}
$$

Here $\Delta^{c o p}=\tau \Delta$ is the comultiplication of the Hopf algebra $H^{c o p}$ and $\tau$ is the switch map.

Now let $A$ be a left $H$-module algebra. It is well-known that there is an induced $H^{o p}$-comodule structure on $A$ as follows:

$$
\chi(a)=\sum R^{(2)} \cdot a \otimes R^{(1)}
$$

It is easy to check that $A$ with (8) is a right $H^{o p}$-comodule algebra. Moreover, $A$ is a Yetter-Drinfel'd $H$-module algebra, for

$$
\begin{aligned}
\chi(h \cdot a) & =\sum R^{(2)} h \cdot a \otimes R^{(1)} \\
& =\sum R^{(2)} h_{(3)} \cdot a \otimes R^{(1)} h_{(2)} S^{-1}\left(h_{(1)}\right) \\
& =\sum h_{(2)} R^{(2)} \cdot a \otimes h_{(3)} R^{(1)} S^{-1}\left(h_{(1)}\right)
\end{aligned}
$$


1.3. Coquasitriangular Hopf algebras. A coquasitriangular Hopf algebra is a pair $(H, \mathcal{R})$, where $H$ is a Hopf algebra with bijective antipode, and $\mathcal{R} \in(H \otimes H)^{*}$ is convolution invertible and satisfies the following axioms:

(CQT1). $\quad \mathcal{R}(h \otimes 1)=\mathcal{R}(1 \otimes h)=\varepsilon(h) 1_{H}$

(CQT2). $\quad \mathcal{R}(a b \otimes c)=\sum \mathcal{R}\left(a \otimes c_{(1)}\right) \mathcal{R}\left(b \otimes c_{(2)}\right)$

(CQT3). $\quad \mathcal{R}(a \otimes b c)=\sum \mathcal{R}\left(a_{(1)} \otimes b\right) \mathcal{R}\left(a_{(2)} \otimes c\right)$

(CQT4). $\quad \sum b_{(1)} a_{(1)} \mathcal{R}\left(a_{(2)} \otimes b_{(2)}\right)=\sum \mathcal{R}\left(a_{(1)} \otimes b_{(1)}\right) a_{(2)} b_{(2)}$

Let $A$ be a right $H^{o p}$-comodule algebra. There is an induced left $H$-module structure on $A$ defined as follows:

$$
h \cdot a=\sum a_{(0)} \mathcal{R}\left(h \otimes a_{(1)}\right)
$$

for all $a \in A, h \in H$. It is easy to check that the $H^{o p}$-comodule algebra $A$ furnished with (9) is a Yetter-Drinfel'd $H$-module algebra.

1.4. Braided tensor categories. A braided tensor category $C$ is a monoidal category equipped with a naturally isomorphic transformation $\Psi$ from the product $\otimes$ to the twisted product $\tau \circ \otimes$ such that

$$
\Psi_{X \otimes Y, Z}=\left(X \otimes \Psi_{Y, Z}\right)\left(\Psi_{X, Z} \otimes Y\right) \text { and } \Psi_{X, Y \otimes Z}=\left(\Psi_{X, Y} \otimes Z\right)\left(Y \otimes \Psi_{X, Z}\right)
$$

$\Psi$ is called the braiding of the category $C$, cf. $[16,27]$. Let $H$ be a Hopf algebra. Denote by $\mathcal{Q}^{H}$ the category of all Yetter-Drinfel'd $H$-modules. Then $\left(\mathcal{Q}^{H}, \tilde{\otimes}, \Psi, k\right)$ forms a braided tensor category cf. $[8,27]$. The notation $\tilde{\otimes}$ for the tensor product indicates that the $H$-structures of $M \tilde{\otimes} N$ comes from both $M$ and $N$, that is

$$
h \cdot(m \tilde{\otimes} n)=\sum h_{(1)} \cdot m \tilde{\otimes} h_{(2)} \cdot n \text { and } \chi(m \tilde{\otimes} n)=\sum m_{(0)} \tilde{\otimes} n_{(0)} \otimes n_{(1)} m_{(1)}
$$

The braiding $\Psi$ is given by

$$
\Psi_{X, Y}: X \tilde{\otimes} Y \longrightarrow Y \tilde{\otimes} X, x \tilde{\otimes} y \mapsto \sum y_{(0)} \tilde{\otimes} y_{(1)} \cdot x
$$

The inverse $\Psi^{-1}$ of $\Psi$ is given by the formula

$$
\Psi^{-1}(y \tilde{\otimes} x)=\sum S\left(y_{(1)}\right) \cdot x \tilde{\otimes} y_{(0)}
$$

If $H$ is a quasitriangular Hopf algebra, then $\mathcal{Q}^{H}$ contains a braided tensor subcategory ${ }_{H} \mathbf{M}$, consisting of all left $H$-modules. From (8) it follows easily that the braiding in ${ }_{H} \mathbf{M}$ is given by

$$
\Psi_{M, N}: M \tilde{\otimes} N \longrightarrow N \tilde{\otimes} M, m \tilde{\otimes} n \mapsto \sum R^{(2)} \cdot n \tilde{\otimes} R^{(1)} \cdot m
$$

Dually, if $H$ is a coquasitriangular Hopf algebra, then the braided tensor category $\mathbf{M}^{H^{o p}}$ of all right $H^{o p}$-comodules is a braided tensor subcategory of $\mathcal{Q}^{H}$.

Let $\mathcal{A}(H)$ denote the category of all Yetter-Drinfel'd $H$-module algebras and Yetter-Drinfel'd $H$-module algebra homomorphisms. Then $(\mathcal{A}(H), \#, k)$ forms a monoidal category. Here \# is the braided product given by

$$
(a \# b)(c \# d)=\sum a b_{(0)} \#\left(b_{(1)} \cdot c\right) d \in A \# B
$$

If $H$ is a quasitriangular Hopf algebra, then the category $\mathcal{A} m(H)$ of all left $H$ module algebras is a submonoidal category of $\mathcal{A}(H)$. Dually, in case $H$ is a coquasitriangular Hopf algebra then the category $\mathcal{A} c(H)$ of all right $H^{o p}$-comodule algebras is a submonoidal category of $\mathcal{A}(H)$. 
1.5. The Brauer group of a Hopf algebra. In [8] we defined the Brauer group of a Hopf algebra $H$ by considering isomorphism classes of $H$-Azumaya algebras. Let $M$ be a Yetter-Drinfel'd $H$-module. $M$ is said to be of finite type if $M$ is finitely generated and projective as a $k$-module. If, in addition, $M$ is faithful over $k$, then $M$ is said to be faithfully projective. For a faithfully projective Yetter-Drinfel'd $H$-module $M$ the endomorphism $\operatorname{ring} \operatorname{End}_{k}(M)$ and the opposite $\operatorname{End}_{k}(M)^{o p}$ are Yetter-Drinfel'd $H$-module algebras, cf. [8]. A Yetter-Drinfel'd $H$-module algebra $A$ is called $H$-Azumaya if it is faithfully projective as a $k$-module and if the following Yetter-Drinfel'd $H$-module algebra maps are isomorphisms:

$$
\begin{aligned}
& F: \quad A \# \bar{A} \longrightarrow \operatorname{End}(A), \quad F(a \# \bar{b})(c)=\sum a c_{(0)}\left(c_{(1)} \cdot b\right) \\
& G: \quad \bar{A} \# A \longrightarrow \operatorname{End}(A)^{o p}, \quad G(\bar{a} \# b)(c)=\sum a_{(0)}\left(a_{(1)} \cdot c\right) b
\end{aligned}
$$

Two $H$-Azumaya algebras $A$ and $B$ are Brauer equivalent (denoted $A \sim B$ ) if there exist two faithfully projective Yetter-Drinfel'd $H$-modules $M$ and $N$ such that $A \# \operatorname{End}(M) \cong B \# \operatorname{End}(N)$. The Brauer group of the Hopf algebra $H$ is denoted by $\mathrm{BQ}(k, H)$.

Now let $(H, R)$ be a quasitriangular Hopf algebra. Since ${ }_{H} \mathbf{M}$ is closed in $\mathcal{Q}^{H}$ under the product $\tilde{\otimes}$ and $\mathcal{A} m(H)$ is closed in $\mathcal{A}(H)$ under the braided product \#, we may define the $\operatorname{Brauer}$ group $\operatorname{BM}(k, H, R)$ of $H$-module algebras. $\operatorname{BM}(k, H, R)$ is the subgroup of $\mathrm{BQ}(k, H)$ consisting of classes of algebras represented by the $H$-Azumaya algebras with comodule structure are of the form (8). We now have the following inclusions:

$$
\mathrm{Br}(k) \subseteq \mathrm{BM}(k, H, R) \subseteq \mathrm{BQ}(k, H)
$$

Dually, for a coquasitriangular Hopf algebra we may define the Brauer group $\mathrm{BC}(k, H, \mathcal{R})$ of right $H^{o p}$-comodule algebras. We then have the following inclusions:

$$
\mathrm{Br}(k) \subseteq \mathrm{BC}(k, H, \mathcal{R}) \subseteq \mathrm{BQ}(k, H)
$$

Now let us look at the finite case. Suppose that $H$ is a faithfully projective Hopf algebra. Then the Drinfel'd double $\mathrm{D}(H)$ is a quasitriangular Hopf algebra, and there is a one-to-one correspondence between left $\mathrm{D}(H)$-module algebras and YetterDrinfel'd $H$-module algebras, cf. [17]. It follows that $\mathrm{BQ}(k, H)=\mathrm{BM}(k, \mathrm{D}(H))$ and, a fortiori, that

$$
\mathrm{BQ}(k, H) \subseteq \mathrm{BQ}(k, \mathrm{D}(H))
$$

Now write $\mathrm{D}^{n}(H)$ for $\mathrm{D}\left(\mathrm{D}^{n-1}(H)\right)$, the $n$-th Drinfel'd double. Then we have the following chain of inclusions:

$$
\mathrm{BQ}(k, H) \subseteq \mathrm{BQ}(k, \mathrm{D}(H)) \subseteq \mathrm{BQ}\left(k, \mathrm{D}^{2}(H)\right) \subseteq \cdots \subseteq \mathrm{BQ}\left(k, \mathrm{D}^{n}(H)\right) \subseteq \cdots
$$

When is the foregoing ascending chain finite?

It would also be interesting to compute the Brauer group of Sweedler's 4-dimensional Hopf algebra $W=k\langle g, x\rangle /\left(g^{2}=1, x^{2}=0, x g=-g x\right)$, which is both a quasitriangular and a coquasitriangular Hopf algebra. In any case, we have the following inclusion diagram:

$$
\begin{array}{ccc}
\operatorname{Br}(k) & \subset & \operatorname{BM}(k, W, R) \\
\cap & & \cap \\
\operatorname{BC}(k, W, \mathcal{R}) & \subset & \operatorname{BQ}(k, W)
\end{array}
$$


Here $\mathrm{BC}(k, W, \mathcal{R}) \simeq \operatorname{BM}(k, W, R)$, because $W$ is self-dual. If $H$ is a commutative and cocommutative Hopf algebra, the Brauer-Long group $\mathrm{BD}(k, H)$ of $H$-dimodule algebras (cf. [14]) is anti-isomorphic to $\mathrm{BQ}(k, H)$ (cf. [8]). Let $H$ be a faithfully projective Hopf algebra, and $\theta: H \rightarrow H^{*}$ a Hopf algebra map. Orzech [19] defined a subgroup $\mathrm{B}_{\theta}(k, H)$ of $\mathrm{BD}(k, H)$ consisting of classes of $\theta$-dimodule algebras. We will show that this is equivalent to defining a coquasitriangular structure $\mathcal{R}$ on $H$ and considering $\mathrm{BC}(k, H, \mathcal{R})$. Let $H$ be a faithfully projective Hopf algebra. Denote by $\mathrm{P}(H, k)$ all the invertible elements in the convolution algebra $(H \otimes H)^{*}$ satisfying (CQT1-CQT3). It is easy to see that $\mathrm{P}(H, k)$ is a group with respect to the convolution product. Since a bialgebra map between Hopf algebras is automatically a Hopf algebra map, we have

Lemma 1.1. ([22]) Let $H$ be a faithfully projective Hopf algebra. Then

$$
\mathrm{P}(H, k) \cong \operatorname{Hopf}\left(H^{o p}, H^{*}\right)
$$

For a commutative and cocommutative faithfully projective Hopf algebra $H$, the axiom CQT4 is automatically satisfied. Therefore each element in $\mathrm{P}(H, k)$ determines a coquasitriangular structure on $H$. We summarize the above arguments as follows:

Lemma 1.2. Let $H$ be a commutative and cocommutative faithfully projective Hopf algebra.

1. There is a one to one correspondence between the coquasitriangular structures on $H$ and the group $\operatorname{Hopf}\left(H, H^{*}\right)$;

2. $\mathrm{B}_{\theta}(k, H)$ is anti-isomorphic to $\mathrm{BC}(k, H, \mathcal{R})$, where $\theta$ corresponds to $\mathcal{R}$.

In particular, the Brauer-Long group $\mathrm{BD}(k, H)$ is anti-isomorphic to the Brauer group $\mathrm{BM}(k, \mathrm{D}(H), R)$ (or $\mathrm{BC}\left(k, \mathrm{D}(H)^{*}, \mathcal{R}\right)$ ), where $R$ and $\mathcal{R}$ are represented by the dual pair of bases for $H$ and $H^{*}$ cf. [12, 22]. Moreover, if $H=k G$ is a finite abelian group algebra, $\phi: G \times G \longrightarrow \mathbb{G}_{m}(k)$ a bilinear map, we may view $\phi$ as a coquasitriangular structure on $H$. Then the Brauer group $\mathrm{B}_{\phi}(k, G)$ of graded algebras (cf. $[9,10])$ is anti-isomorphic to $\mathrm{BC}(k, H, \phi)$.

We claim that a cocommutative Hopf algebra with a coquasitriangular structure is necessarily commutative. Indeed, if $(H, \mathcal{R})$ is such a Hopf algebra, then for $a, b \in H$, CQT4 may be restated as follows: for all $a, b \in H$ :

$$
\sum \mathcal{R}\left(a_{(1)} \otimes b_{(1)}\right) a_{(2)} b_{(2)}=\mathcal{R}\left(a_{(1)} \otimes b_{(1)}\right) b_{(2)} a_{(2)}
$$

since $H$ is cocomutative. It follows that

$$
\begin{aligned}
a b & =\mathcal{R}^{-1}\left(a_{(1)} \otimes b_{(1)}\right) \mathcal{R}\left(a_{(2)} \otimes b_{(2)}\right) a_{(3)} b_{(3)} \\
& =\mathcal{R}^{-1}\left(a_{(1)} \otimes b_{(1)}\right) \mathcal{R}\left(a_{(2)} \otimes b_{(2)}\right) b_{(3)} a_{(3)} \\
& =b a
\end{aligned}
$$

Similarly, a commutative Hopf algebra endowed with a quasitriangular structure is cocommutative.

\section{The Strong Part of BQ $(k, H)$}

2.1. Hopf Yetter-Drinfel'd modules. Let $H$ be a Hopf algebra, and let $A$ be a Yetter-Drinfel'd $H$-module algebra. $\bar{A}$ is the $H$-opposite of $A$. We will call the Yetter-Drinfel'd $H$-module algebra $A^{\# e}=A \# \bar{A}$ (resp. ${ }^{\# e} A=\bar{A} \# A$ ) the left (resp. 
right) $H$-enveloping algebra of $A$. $A$ may be regarded as a left $A^{\# e}$-module and a right ${ }^{\#} A$-module as follows:

$$
(a \# \bar{b}) \cdot x=\sum a x_{(0)}\left(x_{(1)} \cdot b\right) \text { and } x \cdot(\bar{a} \# b)=\sum a_{(0)}\left(a_{(1)} \cdot x\right) b
$$

Let $A$ be a Yetter-Drinfel'd module algebra. Recall that a (left) Hopf YetterDrinfel' $d(A, H)$-module is a $k$-module $M$ which is at once a Yetter-Drinfel' $d H$ module and a left $A$-module satisfying the following compatibility relations:

$$
\begin{aligned}
h \cdot(a m) & =\sum\left(h_{(1)} \cdot a\right)\left(h_{(2)} \cdot m\right) \\
\chi(a m) & =\sum a_{(0)} m_{(0)} \otimes m_{(1)} a_{(1)}
\end{aligned}
$$

A Hopf Yetter-Drinfel'd $H$-module $M$ is a left $A \# H$-module and a right Hopf module in ${ }_{A} \mathbf{M}^{H^{o p}}$. Here $A \# H$ is the usual smash product rather than the braided product. Denote by ${ }_{A} \mathcal{Q}^{H}$ the category of Hopf Yetter-Drinfel'd $(A, H)$-modules and Hopf Yetter-Drinfel'd $(A, H)$-module homomorphisms. Similarly, we may define right Hopf Yetter-Drinfel'd $(A, H)$-modules and the category $\mathcal{Q}_{A}^{H}$ of right Hopf Yetter-Drinfel'd $(A, H)$-modules by imposing the following compatibility relations:

$$
\begin{aligned}
h \cdot(m a) & =\sum\left(h_{(1)} \cdot m\right)\left(h_{(2)} \cdot a\right) \\
\chi(m a) & =\sum m_{(0)} a_{(0)} \otimes a_{(1)} m_{(1)}
\end{aligned}
$$

Let $A, B$ be Yetter-Drinfel'd $H$-module algebras. An $(H, A$-B)-bimodule $M$ is an ( $A$-B)-bimodule which belongs to ${ }_{A} \mathcal{Q}^{H}$ and $\mathcal{Q}_{B}^{H}$. Denote by ${ }_{A} \mathcal{Q}_{B}^{H}$ the category of ( $H, A$ - $B$ )-bimodules. View $k$ as a trivial Yetter-Drinfel'd $H$-module algebra. Then ${ }_{A} \mathcal{Q}^{H}$ is just the category of $(H, A-k)$-bimodules. Similarly $\mathcal{Q}_{A}^{H}$ is the category of $(H, k$ - $A)$-bimodules.

Proposition 2.1. Let $A$ be a Yetter-Drinfel'd $H$-module algebra. Then

1. $\left({ }_{A} \mathcal{Q}_{A}^{H}, \tilde{\otimes}_{A}, A\right)$ is a monoidal category;

2. ${ }_{A} \mathcal{Q}_{A}^{H}$ is equivalent to ${ }_{A}{ }^{*} \mathcal{Q}^{H}$ and $\mathcal{Q}_{\# e}^{H}$;

3. If $A$ is $H$-commutative then ${ }_{A} \mathcal{Q}^{H}$ is a monoidal subcategory of ${ }_{A} \mathcal{Q}_{A}^{H}$.

Proof. 1) is trivial and 3) follows from [8, (3.2.3)]. It only remains to show 2). Define a functor

$$
\kappa:{ }_{A}^{\# e} \mathcal{Q}^{H} \longrightarrow{ }_{A} \mathcal{Q}_{A}^{H}, M \mapsto \kappa(M)
$$

where $\kappa(M)=M$ as a Yetter-Drinfel'd $H$-module but with $A$-bimodule structure given by

$$
a m=(a \# 1) m \text { and } m a=\sum\left(1 \# S\left(m_{(1)}\right) \cdot \bar{a}\right) m_{(0)}
$$

for $a \in A, m \in M$. It is easy to check that $\kappa$ is well-defined and preserves homomorphisms. On the other hand, we have a functor

$$
\iota:{ }_{A} \mathcal{Q}_{A}^{H} \longrightarrow{ }_{A \# e} \mathcal{Q}^{H}
$$

defined as follows : for an $(H, A$-A)-bimodule $M$, let $\iota(M)$ be equal to $M$ as a Yetter-Drinfel'd $H$-module with $A^{\# e}$-module structure defined by

$$
(a \# \bar{b}) m=a\left(m_{(0)}\left(m_{(1)} \cdot b\right)\right)
$$

for $a, b \in A, m \in M$. Observe that $\kappa$ and $\iota$ are each other's inverses. A similar argument leads to the equivalence between ${ }_{A} \mathcal{Q}_{A}^{H}$ and $\mathcal{Q}_{\# e}^{H}$.

It follows from the above proposition that the categories ${ }_{A}^{\# e} \mathcal{Q}^{H}$ and $\mathcal{Q}_{\# e}^{H}$ are equivalent monoidal categories. 


\section{2. $H$-Morita contexts.}

Definition 2.2. A (strict) Morita context $(A, B, P, Q, \varphi, \psi)$ is called a (strict) $H$ Morita context if the following conditions hold:

1. $A, B$ are Yetter-Drinfel'd $H$-module algebras;

2. $P$ is an $(H, A-B)$-bimodule and $Q$ is an $(H, B-A)$-bimodule;

3. $\phi: P \tilde{\otimes}_{B} Q \longrightarrow A$ and $\psi: Q \tilde{\otimes}_{A} P \longrightarrow B$ are (surjective) Yetter-Drinfel'd $H$-module maps.

In the sequel, we list some useful properties. We leave it to the reader to verify that all maps occurring in the next three propositions are Yetter-Drinfel'd $H$ module maps.

Proposition 2.3. For any faithfully projective Yetter-Drinfel'd H-module $P$, we have a strict $H$-Morita context $\left(\operatorname{End}(P), k, P, P^{*}, \varphi, \psi\right)$. The maps $\varphi$ and $\psi$ are given by the formulas

$$
\varphi(p \otimes f)(x)=p f(x) \text { and } \psi(f \otimes p)=f(p)
$$

Let $B$ be a Yetter-Drinfel'd $H$-module algebra and $P \in \mathcal{Q}_{B}^{H}$ be a finitely generated projective right $B$-module. Then $\operatorname{End}_{B}(P)$ is a Yetter-Drinfel'd $H$-module algebra with $H$-structures given by (4) and (5). If $Q$ is a finitely generated projective left $B$-module which is in ${ }_{B} \mathcal{Q}^{H}$, then $\operatorname{End}_{B}(Q)$ is a Yetter-Drinfel'd $H$-module algebra with $H$-structure given by (6) and (7). Note that $\operatorname{End}_{B}(Q)$ acts on $Q$ on the right hand side.

Proposition 2.4. Let $B$ be a Yetter-Drinfel'd $H$-module algebra. If $P \in \mathcal{Q}_{B}^{H}$ is a B-progenerator, then $\left(A=\operatorname{End}_{B}(P), B, P, Q=\operatorname{Hom}_{B}(P, B), \varphi, \psi\right)$ is a strict $H$-Morita context. Here $\varphi$ and $\psi$ are given by

$$
\varphi(p \otimes f)(x)=p f(x) \text { and } \psi(f \otimes p)=f(p)
$$

Proposition 2.5. Let $(A, B, P, Q, \varphi, \psi)$ be a strict $H$-Morita context. Then the pairs of functors

$$
\begin{aligned}
& Q \tilde{\otimes}_{A} \bullet:{ }_{A} \mathcal{Q}^{H} \longrightarrow{ }_{B} \mathcal{Q}^{H} \quad \text { and } P \tilde{\otimes}_{B} \bullet:{ }_{B} \mathcal{Q}^{H} \longrightarrow{ }_{A} \mathcal{Q}^{H} \\
& \bullet \tilde{\otimes}_{A} P: \mathcal{Q}_{A}^{H} \longrightarrow \mathcal{Q}_{B}^{H} \text { and } \bullet \tilde{\otimes}_{B} Q: \mathcal{Q}_{B}^{H} \longrightarrow \mathcal{Q}_{A}^{H}
\end{aligned}
$$

define equivalences between categories of Hopf Yetter-Drinfel'd H-modules.

Now substituting $A^{\# e}$ or ${ }^{\# e} A$ for $A$, we obtain the categories ${ }_{A \# e} \mathcal{Q}^{H}$ and $\mathcal{Q}_{\# e}^{H}$. The equation (12) tells us that $A$ is an object ${ }_{A \# e} \mathcal{Q}^{H}$ and $\mathcal{Q}_{\# \text { e }}^{H}$. To an Hopf Yetter-

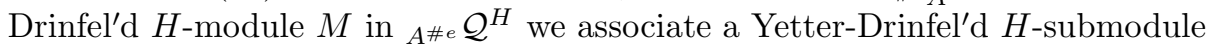

$$
M^{A}=\{m \in M \mid(a \# 1) m=(1 \# \bar{a}) m, \forall a \in A\}
$$

In the sequel, we will use the shorter notations $a m$ for $(a \# 1) m$ and $\bar{a} m$ for $(1 \# \bar{a}) m$. $M^{A}$ is a Yetter-Drinfel'd $H$-module since for $h \in H, a \in A$ and $m \in M$,

$$
\begin{aligned}
a(h \cdot m) & =\sum h_{(2)}\left(S^{-1}\left(h_{(1)}\right) \cdot a\right) m \\
& =\sum h_{(2)}\left(S^{-1}\left(h_{(1)}\right) \cdot \bar{a}\right) m=\bar{a}(h \cdot m)
\end{aligned}
$$

and

$$
\begin{aligned}
(a \otimes 1) \chi(m) & =\sum a_{(0)} m_{(0)} \otimes m_{(1)} a_{(1)} S\left(a_{(2)}\right)=\sum \chi\left(a_{(0)} m\right)\left(1 \otimes S\left(a_{(1)}\right)\right) \\
& =\sum \chi\left(\overline{a_{(0)}} m\right)\left(1 \otimes S\left(a_{(1)}\right)\right)=(\bar{a} \otimes 1) \chi(m)
\end{aligned}
$$


We therefore have a functor $(\bullet)^{A}$ from ${ }_{A} \# \mathcal{Q}^{H}$ to $\mathcal{Q}^{H}$. On the other hand, we have an induction functor $A \tilde{\otimes} \bullet$ from $\mathcal{Q}^{H}$ to ${ }_{A \# e} \mathcal{Q}^{H}$. It is easy to see that

$$
\begin{aligned}
& A \tilde{\otimes} \bullet: \quad \mathcal{Q}^{H} \quad \longrightarrow \quad A_{A{ }^{\# e}} \mathcal{Q}^{H}, \quad N \mapsto A \tilde{\otimes} N \\
& (\bullet)^{A}: \quad{ }_{A \# e} \mathcal{Q}^{H} \longrightarrow \mathcal{Q}^{H}, \quad M \mapsto M^{A}
\end{aligned}
$$

is a pair of adjoint functors. Similarly we have a pair of adjoint functors between the categories $\mathcal{Q}^{H}$ and $\mathcal{Q}_{\# e}^{H}$ :

$$
\begin{aligned}
& \bullet \tilde{\otimes} A: \quad \mathcal{Q}^{H} \quad \longrightarrow \quad \mathcal{Q}_{\# e}^{H}, \quad N \mapsto N \tilde{\otimes} A \\
& { }^{A}(\bullet): \quad \mathcal{Q}_{\# e_{A}}^{H} \longrightarrow \mathcal{Q}^{H}, \quad M \mapsto{ }^{A} M=\{m \in M \mid m a=m \bar{a}, \forall a \in A\}
\end{aligned}
$$

Proposition 2.6. Let $A$ be an $H$-Azumaya algebra. Then (17) and (18) define equivalences of categories.

Proof. $A$ is $H$-Azumaya, so $A$ is a faithfully projective Yetter-Drinfel'd $H$-module, $A^{\# e} \cong \operatorname{End}(A)$ and ${ }^{\# e} A \cong \operatorname{End}(A)^{o p}$. It follows from (2.3) and (2.4) that the pair of functors $\left(A \tilde{\otimes} \bullet, A^{*} \tilde{\otimes}_{A \# \bullet} \bullet\right)$ defines an equivalence between $\mathcal{Q}^{H}$ and ${ }_{A_{A+}} \mathcal{Q}^{H}$. However $\left(A \tilde{\otimes} \bullet,(\bullet)^{A}\right)$ is an adjoint pair of functors. By the uniqueness of adjoint functors, $(\bullet)^{A}$ is isomorphic to $A^{*} \tilde{\otimes}_{A \# \bullet} \bullet$. A similar argument applies to (18).

In fact, (17) and (18) define equivalences between braided tensor categories if $A$ is an $H$-Azumaya algebra.

Proposition 2.7. Let $A$ be an $H$-Azumaya algebra. Then the category ${ }_{A} \mathcal{Q}_{A}^{H}$ is a braided tensor category, and the following pair of monoidal functors:

$$
\begin{aligned}
& A \tilde{\otimes} \bullet: \quad \mathcal{Q}^{H} \quad \longrightarrow \quad{ }_{A} \mathcal{Q}_{A}^{H}, \quad N \mapsto A \tilde{\otimes} N \\
& (\bullet)^{A}:{ }_{A} \mathcal{Q}_{A}^{H} \longrightarrow \mathcal{Q}^{H}, \quad M \mapsto M^{A},
\end{aligned}
$$

where $M^{A}=\left\{m \in M \mid a m=\sum m_{(0)}\left(m_{(1)} \cdot a\right), \forall a \in A\right\}$, defines an equivalence of braided tensor categories, where the induced $(H, A-A)$-bimodule $A \tilde{\otimes} N$ of $N$ has the left $A$-module structure coming from that of $A$, and has the right $A$-module structure given by $(b \otimes m) a=\sum b\left(S\left(m_{(1)}\right) \cdot a\right) \otimes m_{(0)}$ for $b \otimes m \in A \tilde{\otimes} M$ and $a \in A$.

Proof. By (2.6) the pair of functors (19) defines an equivalence. If $A \tilde{\otimes} \bullet$ is a monoidal functor, then the braiding on the category $\mathcal{Q}^{H}$ induces a braiding on the category ${ }_{A} \mathcal{Q}_{A}^{H}$ and the statements follow. For Yetter-Drinfel'd $H$-modules $M$ and $N$, it is not hard to check that we have the following isomorphism in ${ }_{A} \mathcal{Q}_{A}^{H}$ :

$$
A \tilde{\otimes}(M \tilde{\otimes} N) \simeq(A \tilde{\otimes} M) \tilde{\otimes}_{A}(A \tilde{\otimes} N)
$$

Also if $f, g$ are homomorphisms in $\mathcal{Q}^{H}$ then $A \tilde{\otimes}(f \tilde{\otimes} g) \simeq(A \tilde{\otimes} f) \tilde{\otimes}_{A}(A \tilde{\otimes} g)$. Therefore $A \tilde{\otimes} \bullet$ is a monoidal functor.

We will now show that Brauer equivalence of $H$-Azumaya algebras is equivalent to $H$-Morita equivalence. We write $A \stackrel{m}{\sim} B$ if $A$ and $B$ are $H$-Morita equivalent, and $A \stackrel{b}{\sim} B$ if $A$ and $B$ are Brauer equivalent, that is, $[A]=[B] \in \mathrm{BQ}(k, H)$.

Lemma 2.8. Let $A, B$ and $C$ be Yetter-Drinfel' $d H$-module algebras. If $A \stackrel{m}{\sim} B$, then $A \# C \stackrel{m}{\sim} B \# C$ and $C \# A \stackrel{m}{\sim} C \# B$. 
Proof. Suppose that $\{A, B, P, Q,[],,()$,$\} is a strict H$-Morita context. Write $\bar{P}=$ $P \tilde{\otimes} C$ and $\bar{Q}=Q \tilde{\otimes} C$ as Yetter-Drinfel'd $H$-modules. We endow $\bar{P}$ with a left $A \# C$-module and a right $B \# C$-module structure as follows:

$$
\begin{aligned}
& (a \# c)-(p \tilde{\otimes} x)=\sum a p_{(0)} \tilde{\otimes}\left(p_{(1)} \cdot c\right) x \\
& (p \tilde{\otimes} x)-(b \# c)=\sum p b_{(0)} \tilde{\otimes}\left(b_{(1)} \cdot x\right) c
\end{aligned}
$$

for $a \in A, b \in B, c, x \in C$ and $p \in P$. Then $\bar{P}$ is an $(H, A \# C$-B\#C)-bimodule (the tedious check is left to the reader). Similarly, $\bar{Q}$ with the following structures is an $(H, B \# C$ - $A \# C)$-bimodule:

$$
\begin{aligned}
& (b \# c)-(q \tilde{\otimes} y)=\sum b q_{(0)} \tilde{\otimes}\left(q_{(1)} \cdot c\right) y \\
& (q \tilde{\otimes} y)-(a \# c)=\sum q a_{(0)} \tilde{\otimes}\left(a_{(1)} \cdot y\right) c
\end{aligned}
$$

Define the maps

$$
[,]: \bar{P} \tilde{\otimes}_{B \# C} \bar{Q} \longrightarrow A \# C \text { and }(,): \bar{Q} \tilde{\otimes}_{A \# C} \bar{P} \longrightarrow B \# C
$$

by

$$
\begin{aligned}
{[p \tilde{\otimes} x, q \tilde{\otimes} y] } & =\sum\left[p, q_{(0)}\right] \#\left(q_{(1)} \cdot x\right) y \\
(q \tilde{\otimes} y, p \tilde{\otimes} x) & =\sum\left(q, p_{(0)}\right) \#\left(p_{(1)} \cdot y\right) x
\end{aligned}
$$

$[$,$] is well-defined since$

$$
[p \tilde{\otimes} x \leftarrow b \# c, q \tilde{\otimes} y]=\sum\left[p b_{(0)}, q_{(0)}\right] \#\left(q_{(1)} b_{(1)} \cdot x\right)\left(q_{(2)} \cdot c\right) y=[p \tilde{\otimes} x, b \# c-q \tilde{\otimes} y]
$$

for any $b \in B, c, x, y \in C, p \in P$ and $q \in Q$. Similarly (,) is well defined. Both [,] and $($,$) are H$-(co)linear since they are the composite maps of $H$-(co)linear maps and the braiding maps. A similar argument yields that [,] and (, ) are surjective. We leave it to the reader to check that [,] is an $A \# C$-bimodule map and (,) is a $B \# C$ bimodule map, and hence $A \# C \stackrel{m}{\sim} B \# C$. Similarly, we have $C \# A \stackrel{m}{\sim} C \# B$.

Lemma 2.9. If $A \stackrel{m}{\sim} B$, then $\bar{A} \stackrel{m}{\sim} \bar{B}$.

Proof. Suppose that $\{A, B, P, Q,[],,()$,$\} is a strict H$-Morita context. Then the $(H, A$ - $B$ )-bimodule structure on $P$ yields an $(H, \bar{B}-\bar{A})$-bimodule structure on $P$ as follows (P equipped with this $(H, \bar{B}-\bar{A})$-bimodule structure will be denoted by $\bar{P})$ :

$$
\bar{b}-p=\sum p_{(0)}\left(p_{(1)} \cdot b\right), \text { and } p \leftarrow \bar{a}=\sum a_{(0)}\left(a_{(1)} \cdot p\right)
$$

Indeed, for $\bar{a} \in \bar{A}, \bar{b} \in \bar{B}, p \in P$, we have

$$
\begin{aligned}
(\bar{b}-p) \leftarrow \bar{a} & =\sum a_{(0)}\left(a_{(1)} \cdot\left(p_{(0)}\left(p_{(1)} \cdot b\right)\right)\right) \\
& =\sum\left(a_{(0)}\left(a_{(1)} \cdot p_{(0)}\right)\right)\left(a_{(2)} p_{(1)} \cdot b\right) \\
& =\bar{b}-(p-\bar{a})
\end{aligned}
$$

This means that $\bar{P}$ is a $(\bar{B}-\bar{A})$-bimodule. This bimodule structure is compatible with the $H$-(co)module structures on $P$, and thus $\bar{P}$ is a $(H, \bar{B}-\bar{A})$-bimodule. Similarly, interchanging $\bar{A}$ and $\bar{B}$ and substituting $Q$ for $P$, we obtain an $(H, \bar{A}-\bar{B})$-bimodule 
$\bar{Q}$. One may readily verify that the following maps are the desired maps for the strict $H$-Morita context $\left\{\bar{A}, \bar{B}, \bar{Q}, \bar{P},[,]^{\prime},(,)^{\prime}\right\}$ :

$$
\begin{array}{ll}
{[,]^{\prime}:} & \bar{Q} \tilde{\otimes}_{\bar{B}} \bar{P} \longrightarrow \bar{A}, \quad[\bar{q}, \bar{p}]^{\prime}=\sum \overline{\left[p_{(0)}, p_{(1)} \cdot q\right]} \\
(,)^{\prime}: & \bar{P} \tilde{\otimes}_{\bar{A}} \bar{Q} \longrightarrow \bar{B}, \quad(\bar{p}, \bar{q})^{\prime}=\sum \overline{\left(q_{(0)}, q_{(1)} \cdot p\right)}
\end{array}
$$

It follows that $\bar{A} \stackrel{m}{\sim} \bar{B}$.

Theorem 2.10. Let $A, B$ be $H$-Azumaya algebras. $A \stackrel{b}{\sim} B$ if and only if $A \stackrel{m}{\sim} B$.

Proof. Suppose that $A \stackrel{b}{\sim} B$. Then there are faithfully projective Yetter-Drinfel'd $H$-modules $M, N$ such that $A \# \operatorname{End}_{k}(M) \cong B \# \operatorname{End}_{k}(N)$. By Lemma 2.8 we have

$$
A \cong A \# k \stackrel{m}{\sim} A \# \operatorname{End}(M) \stackrel{m}{\sim} B \# \operatorname{End}(N) \stackrel{m}{\sim} B \# k \stackrel{m}{\sim} B
$$

Conversely, if $A \stackrel{m}{\sim} B$, then there is a strict $H$-Morita context $\{A, B, P, Q,[],,()$,$\} .$ Since $Q$ is a faithfully projective Yetter-Drinfel'd $H$-module, $C=\operatorname{End}_{k}(Q)$ is an $H$-Azumaya algebra. We view $B \cong \operatorname{End}_{-A}(Q)$ as an $H$-Azumaya subalgebra of $C$. It follows from (17) that

$$
C=B \tilde{\otimes} C^{B}=B \# C^{B}
$$

We claim that $C^{B}=\operatorname{End}\left(Q_{\bar{B}}\right)$, where $Q_{\bar{B}}=\bar{Q}_{\bar{B}}$. In fact, for $f \in \operatorname{End}\left(Q_{\bar{B}}\right), b \in B$ and $x \in Q$,

$$
\begin{aligned}
f b(x) & =\sum f\left(\left(S\left(b_{(1)}\right) \cdot x\right)-\bar{b}_{(0)}\right) \\
& =\sum f\left(S\left(b_{(1)}\right) \cdot x\right)-\overline{b_{(0)}} \\
& =\sum b_{(0)}\left(b_{(1)} \cdot f\left(S\left(b_{(2)}\right) \cdot x\right)\right) \\
& =\sum b_{(0)}\left(b_{(1)} \cdot f\right)(x)
\end{aligned}
$$

This implies $f b=\sum b_{(0)}\left(b_{(1)} \cdot f\right)$, i.e, $f \in C^{B}$. On the other hand, if $f \in C^{B}$, then for any $x \in Q, b \in B$, we have

$$
\begin{aligned}
f(x \leftarrow \bar{b}) & =\sum f\left(b_{(0)}\left(b_{(1)} \cdot x\right)\right) \\
& =\sum b_{(0)}\left(b_{(1)} \cdot f\right)\left(b_{(2)} \cdot x\right) \\
& =\sum b_{(0)}\left(b_{(1)} \cdot f(x)\right) \\
& =f(x) \leftarrow \bar{b}
\end{aligned}
$$

However, $\bar{A} \cong \operatorname{End}\left(\bar{Q}_{\bar{B}}\right)$ by (3.9). It follows that $B \# \bar{A} \cong B \# C^{B}=\operatorname{End}(Q)$. Therefore,

$$
B \# \operatorname{End}(\bar{A})^{o p} \cong B \# \bar{A} \# A \cong \operatorname{End}(Q) \# A \cong A \# \operatorname{End}(Q)
$$

and hence $A \stackrel{b}{\sim} B$.

Corollary 2.11. If $[A]=1$ in $\mathrm{BQ}(k, H)$, then $A \cong \operatorname{End}(P)$ for some faithfully projective Yetter-Drinfel'd $H$-module $P$.

Proof. By the above theorem $A$ is $H$-Morita equivalent to $k$. 
2.3. $H$-separable algebras. It is well-known, cf. e.g. [13], that a $k$-algebra $A$ is an Azumaya algebra if and only if it is central and separable. Recall that $A$ is separable if and only if $A$ is $A^{e}$-projective, where $A^{e}=A \otimes A^{o p}$. Now we may ask the following question: can we give a similar characterization for $H$-Azumaya algebras? In the case where $H$ is commutative, cocommutative and faithfully projective, this question was investigated by Beattie in [1, Ch. 3]. We call a Yetter-Drinfel'd $H$ module algebra left (resp. right) $H$-separable if it is projective as a left $A^{\#} e_{\text {-module }}$ (resp. a right $\# e A$-module. If $A$ is an $H$-Azumaya algebra, then $A$ is left and right $H$-separable: indeed, in view of the results obtained in the previous section, we have a strict $H$-Morita context $\left(A^{\# e}, k, A, A^{*}, \varphi, \psi\right)$, and therefore $A$ is left $A^{\# e_{-}}$ projective, and $A^{A}=k$ (we will say that $A$ is left $H$-central).

In general, however, we do not have the converse property. We only have the following partial result, due to Beattie (cf. [1, Prop. 3.10]): if $H=k G$, where $G$ is a finite abelian group, with the order of $G$ invertible in $k$, then a left and right $H$-central, left and right $H$-separable Yetter-Drinfel'd $H$-module algebra is an $H$-Azumaya algebra.

In order to have equivalence, we have to replace separability by a stronger property:

Proposition 2.12. Let $A$ be a Yetter-Drinfel'd H-module algebra. Then the following assertions are equivalent:

1. $A$ is an H-Azumaya algebra;

2. A is left and right $H$-central, a left $A^{\# e}$-progenerator and a right ${ }^{\# e} A$-progenerator.

Proof. This follows immediately from the Morita theory developed in the previous section; the argument is identical to the one in the commutative, cocommutative faithfully projective case, cf. [1, Prop. 3.6].

We will now introduce a stronger version of $H$-separability, and this will lead us to the notion of strong $H$-Azumaya algebra.

Definition 2.13. A Yetter-Drinfel'd $H$-module algebra $A$ is called strongly $H$ separable if the exact sequence

$$
A^{\# e} \stackrel{\pi_{A}}{\longrightarrow} A \longrightarrow 0
$$

splits in ${ }_{A \# e} \mathcal{Q}^{H}$.

Here $\pi_{A}$ is the usual multiplication in $A$. It is obvious that a strongly $H$-separable algebra is $H$-separable : if the sequence splits in ${ }_{A}^{\# e} \mathcal{Q}^{H}$, then it certainly splits in $A^{\# e}$-mod.

In the sequel, we often use $M_{0}$ as a shorter notation for $M^{H} \cap M^{c o H}$, the intersection of the invariants and the coinvariants of a Yetter-Drinfel'd $H$-module $M$. In the next proposition, we will see that it does not make sense to distinguish between left and right strong $H$-separability.

Proposition 2.14. Let $A$ be a Yetter-Drinfel'd H-module algebra. The following statements are equivalent:

1. A is H-separable;

2. There exists an element $e_{l} \in A_{0}^{\# e}$ such that $\pi_{A}\left(e_{l}\right)=1$ and $(a \# 1) e_{l}=(1 \# \bar{a}) e_{l}$ for all $a \in A$; 
3. There exists an element $e \in(A \tilde{\otimes} A)_{0}$ such that $\pi_{A}(e)=1$ and $(a \otimes 1) e=$ $e(1 \otimes a)$ for all $a \in A$;

4. There exists an element $e_{r} \in{ }^{\# e} A_{0}$ such that $\pi_{A}\left(e_{r}\right)=1$ and $e_{r}(\bar{a} \# 1)=$ $e_{r}(\overline{1} \# a)$ for all $a \in A$;

5. $\pi_{A}: \bar{A} \# A \longrightarrow A \longrightarrow 0$ splits in $\mathcal{Q}_{\# e_{A}}^{H}$.

Proof. Straightforward.

Note that $H$-separable algebras are $k$-separable by 3 ) of the above proposition (a similar observation is not true for $H$-separable algebras!). Moreover, If $e_{l}=$ $\sum x_{i} \# \bar{y}_{i}$, we may choose $e=\sum x_{i} \otimes y_{i}$ and $e_{r}=\sum \bar{x}_{i} \# y_{i}$. Thus we may write $e_{A}$ for $e_{l}$ and $e_{A}^{\prime}$ for $e_{r}$ without ambiguity. Since $e_{A}$ is an idempotent element in each of the above cases, it follows that if $A$ is strongly $H$-separable then $M^{A}=e_{A} \rightarrow M$ and ${ }^{A} N=N \leftarrow e_{A}^{\prime}$ for $M \in{ }_{A \# e} \mathcal{Q}^{H}$ and $N \in \mathcal{Q}_{\# e}^{H}$ respectively. In particular, $A^{A}=e_{A} \rightarrow A$ and ${ }^{A} A=A \leftarrow e_{A}^{\prime}$. For a Yetter-Drinfel'd $H$-module algebra $A$ we will call $A^{A}$ (resp. ${ }^{A} A$ ) the left (resp. right) $H$-center of $A$. If $A^{A}=k$ (resp. ${ }^{A} A=k$ ) then we call $A$ left (resp. right) $H$-central. $A$ is $H$-central if $A$ is both left and right $H$-central.

Proposition 2.15. 1. Let $f: A \longrightarrow B$ be an epimorphism of Yetter-Drinfel'd $H$-module algebras. If $A$ is strongly $H$-separable, so is $B$.

2. Let $S$ be a commutative $k$-algebra. If $A$ is strongly $H$-separable then $S \otimes_{R} A$ is a strongly $H$-separable $S$-algebra.

Proof. Use the fact that the image of the separability idempotent $e_{A}$ is non-zero under any Yetter-Drinfel'd $H$-module algebra map.

Proposition 2.16. $\quad$ 1. If $A$ is strongly $H$-separable, so is $\bar{A}$. If, in addition, $A$ is left (resp. right) $H$-central then $\bar{A}$ is right (resp. left) $H$-central.

2. If $A, B$ are strongly $H$-separable, then $A \# B$ is strongly $H$-separable. If in addition, $A$ and $B$ are left (resp. right) $H$-central, then $A \# B$ is left (resp. right) $H$-central.

Proof. 1) Let $e_{A}=\sum x_{i} \# \bar{y}_{i}$ be a separability idempotent of $A^{\# e}$. A routine computation shows that

$$
e_{\bar{A}}=\sum \overline{S\left(x_{i(1)}\right) \cdot y_{i}} \# \overline{\overline{x_{i(0)}}}
$$

is a separability idempotent of $\bar{A}^{e}$. It follows that $\bar{A}$ is strongly $H$-separable. Now

$$
\begin{aligned}
\bar{A}_{\bar{A}}=\bar{A} \leftarrow e_{\bar{A}}^{\prime} & =\left\{\sum \overline{S\left(x_{i(1)}\right) \cdot y_{i}} \circ \overline{x_{i(0)}} \mid a \in A\right\} \\
& =\left\{\sum \overline{x_{i} a_{(0)}\left(a_{(1)} \cdot y_{i}\right)} \mid a \in A\right\} \\
& =\overline{A^{A}}
\end{aligned}
$$

This implies that if $A$ is left central then $\bar{A}$ is right central. Similarly, we have $\bar{A}^{\bar{A}}=\overline{{ }^{A} A}$.

2) Write $C$ for $A \# B$, and let $e_{A}=\sum x_{i} \# \overline{y_{i}}, e_{B}=\sum a_{j} \# \overline{b_{j}}$. Set

$$
e=\sum\left(x_{i(0)} \# x_{i(1)} \cdot a_{j}\right) \# \overline{y_{i} \# b_{j}}
$$

We will show that $e$ is a separability idempotent for $C^{e}$. It is easy to see that $e \in C_{0}$ and that

$$
\pi_{C}(e)=\sum x_{i(0)} y_{i(0)} \#\left(y_{i(1)} x_{i(1)} \cdot a_{j}\right) b_{j}=\sum x_{i} y_{i} \# a_{j} b_{j}=1 \# 1 \in C
$$


Take $c=a \# b \in C$. We then have

$$
\begin{aligned}
(c \# \overline{1 \# 1}) e & =\sum a x_{i(0)} \# x_{i(1)} \cdot\left(b a_{j}\right) \# \overline{y_{i} \# b_{j}} \\
& =\sum\left(a x_{i(0)} \# x_{i(1)} \cdot a_{j}\right) \# \overline{y_{i} \# b_{j} b}
\end{aligned}
$$

On the other hand,

$$
\begin{aligned}
(1 \# 1 \# \bar{c}) e & =\sum x_{i(0)} \# x_{i(1)} \cdot a_{j(0)} \# \overline{x_{i(2)} a_{j(1)} \cdot(a \# b)} \circ \overline{y_{i} \# b_{j}} \\
& =\sum x_{i(0)} \# x_{i(1)} \cdot a_{j} \# \overline{\left(y_{i} \# b_{j}\right)(a \# b)} \\
& =\sum x_{i(0)} \# x_{i(1)} \cdot a_{j} \# \overline{y_{i} a_{(0)} \#\left(a_{(1)} \cdot b_{j}\right) b} \\
& =\sum\left(a_{(0)} x_{i}\right)_{(0)} \#\left(a_{(0)} x_{i}\right)_{(1)} \cdot a_{j} \# \overline{y_{i} \#\left(a_{(1)} \cdot b_{j}\right) b} \\
& =\sum a_{(0)} x_{i(0)} \# x_{i(1)} a_{(1)} \cdot a_{j} \# \overline{y_{i} \#\left(a_{(2)} \cdot b_{j}\right) b} \\
& =\sum a x_{i(0)} \# x_{i(1)} \cdot a_{j} \# \overline{y_{i} \# b_{j} b}
\end{aligned}
$$

The last but one equality holds because $e_{B} \in B_{0}^{e}$. It follows that $(c \# \overline{1 \# 1}) e=$ $(1 \# 1 \# \bar{c}) e$ for any $c \in C^{e}$, and hence $C$ is strongly $H$-separable.

Suppose that $A$ and $B$ are right central. Let us show that $C=A \# B$ is also right central. Take $e$ as before. For $a \in A, b \in B$,

$$
\begin{aligned}
(a \# b)-e^{\prime} & =\sum\left(x_{i(0)} \# x_{i(1)} \cdot a_{j(0)}\right)\left(x_{i(2)} a_{j(1)} \cdot a \# x_{i(3)} a_{j(2)} \cdot b\right)\left(y_{i} \# b_{j}\right) \\
& =\sum\left(x_{i(0)} \# x_{i(1)} \cdot a_{j(0)}\right)\left(\left(x_{i(2)} a_{j(1)} \cdot a\right) y_{i(0)} \#\left(y_{i(1)} x_{i(3)} a_{j(2)} \cdot b\right) b_{j}\right) \\
& =\sum x_{i(0)}\left(x_{i(3)} a_{j(2)} \cdot a_{(0)}\right) y_{i(0)} \#\left(y_{i(1)} x_{i(4)} a_{j(3)} a_{(1)} S^{-1}\left(a_{j(1)}\right)\right. \\
\left.\cdot S^{-1}\left(x_{i(2)}\right) x_{i(1)} \cdot a_{j(0)}\right)\left(a_{j(4)} \cdot b\right) b_{j} & \\
& =\sum x_{i(0)}\left(x_{i(1)} a_{j(2)} \cdot a_{(0)}\right) y_{i} \#\left(a_{j(3)} a_{(1)} S^{-1}\left(a_{j(1)}\right) \cdot a_{j(0)}\right)\left(a_{j(4)} \cdot b\right) b_{j}
\end{aligned}
$$

Now, $\sum x_{i(0)}\left(x_{i(1)} a_{j(1)} \cdot a\right) y_{i}=\sum\left(a_{j(1)} \cdot a\right) \leftarrow e_{A}^{\prime} \in k$ implies that

$$
\begin{aligned}
\chi\left(\sum x_{i(0)}\left(x_{i(1)} a_{j(1)} \cdot a\right) y_{i}\right) & =\sum x_{i(0)}\left(x_{i(1)} a_{j(2)} \cdot a_{(0)}\right) y_{i} \otimes a_{j(3)} a_{(1)} S^{-1}\left(a_{j(1)}\right) \\
& =\sum x_{i(0)}\left(x_{i(1)} a_{j(1)} \cdot a\right) y_{i} \otimes 1
\end{aligned}
$$

It follows that

$$
\begin{aligned}
(a \# b)-e^{\prime} & =\sum x_{i(0)}\left(x_{i(1)} a_{j(1)} \cdot a\right) y_{i} \# a_{j(0)}\left(a_{j(2)} \cdot b\right) b_{j} \\
& =\sum 1 \# a_{j(0)}\left(a_{j(2)} \cdot b\right) x_{i(0)}\left(x_{i(1)} a_{j(1)} \cdot a\right) y_{i} b_{j} \in k \# B .
\end{aligned}
$$

Now let $l=\sum \overline{\left(1 \# a_{j}\right)} \#\left(1 \# b_{j}\right)$. We have the identities

$$
\begin{aligned}
e^{\prime} l & \left.=\sum e^{\prime} \overline{\left[\left(\# a_{j}\right)\right.} \#(1 \# 1)\right]\left[\overline{(1 \# 1)} \#\left(1 \# b_{j}\right)\right] \\
& \left.=\sum e^{\prime} \overline{(1 \# 1)} \#\left(1 \# a_{j}\right)\right]\left[\overline{(1 \# 1)} \#\left(1 \# b_{j}\right)\right] \\
& \left.=\sum e^{\prime} \overline{(1 \# 1)} \#\left(1 \# a_{j} b_{j}\right)\right] \\
& =e^{\prime}
\end{aligned}
$$

Denote by $1 \# d$ the element $(a \# b) \longleftarrow e^{\prime} \in k \# B$. Then we have

$$
1 \# d=(a \# b) \leftarrow e^{\prime} l=(1 \# d) \leftarrow l=1 \# d \leftarrow e_{B}^{\prime} \in k \# k
$$


We therefore have that ${ }^{C} C=C \longleftarrow e^{\prime}=k \# k=k$, and it follows that $C$ is right $H$-central. The statement about the left central property follows from the equality

$$
\begin{aligned}
e \rightarrow C & =\bar{C}-\bar{e}=(\bar{B} \# \bar{A})-\bar{e} \\
& ={ }^{\bar{B}} \bar{B} \#^{\bar{A}} \bar{A}=k \# k=k
\end{aligned}
$$

Definition 2.17. A Yetter-Drinfel'd $H$-module algebra $A$ is called (left, right) strongly $H$-Azumaya if $A$ is strongly $H$-separable and (left, right) $H$-central.

In the sequel, we will show that strongly $H$-Azumaya algebras are $H$-Azumaya algebras, justifying our terminology.

Lemma 2.18. Let $A$ be left or right strongly $H$-Azumaya. Then the inclusion map embeds $k$ as a direct summand of $A$ in $\mathcal{Q}^{H}$.

Proof. Let $e_{A}$ be the separability idempotent of $A^{\# e}$. Define a map $t: A \longrightarrow A$ by $t(x)=e_{A} \rightarrow x$ (or $t(x)=x \leftarrow e_{A}^{\prime}$ ). It is easy to see that $t$ is $H$-linear and $H$-colinear, and hence $t$ is a section of the inclusion map in $\mathcal{Q}^{H}$.

Definition 2.19. A Yetter-Drinfel'd $H$-module algebra $A$ has an $H$-trace map if the inclusion map $i: k \hookrightarrow A$ has a section in $\mathcal{Q}^{H}$. A Yetter-Drinfel'd $H$-module algebra $A$ is said to be $H$-simple if $A$ has no proper Yetter-Drinfel'd $H$-module ideal (simply $H$-ideal). This is equivalent to $A$ being simple in ${ }_{A \# e} \mathcal{Q}^{H}$ or $\mathcal{Q}_{\# e}^{H}$.

Proposition 2.20. Let $A$ be a left (or right) strongly $H$-Azumaya algebra. Then $A$ is $H$-simple if and only if $k$ is a field.

Proof. Suppose that $A$ is $H$-simple and $I$ is a non-zero ideal of $k$. $I A$ is an $H$-ideal of $A$, and $I A=A$. Let $t$ be the $H$-trace map described in Lemma 2.18. Then $t(I A)=t(A)$ implies $I=k$. It follows that $k$ is a field.

Conversely, suppose that $A$ is a strongly $H$-separable algebra over a field $k$. Since $H$-separability implies $k$-separability, $A$ is semisimple artinian. Let $M$ be an $H$ ideal of $A$. Then there exists a central idempodent $c \in A$ such that $M=c A=A c$. $c$ must be in $A_{0}$. In fact, for $h \in H$ (note that $\forall m \in M, c m=m=m c$ and $c \in M$ )

$$
\begin{aligned}
h \cdot c & =(h \cdot c) c=\sum\left(h_{(1)} \cdot c\right)\left(\varepsilon\left(h_{(2)}\right) c\right) \\
& =\sum h_{(1)}\left(c\left(S\left(h_{(2)}\right) \cdot c\right)\right) \\
& =\sum h_{(1)} \cdot\left(S\left(h_{(2)}\right) \cdot c\right)=\varepsilon(h) c
\end{aligned}
$$

Since $c$ is in $\mathcal{M}$ we have $\sum c c_{(0)} \otimes c_{(1)}=\sum c_{(0)} \otimes c_{(1)}$. Therefore $\left(c=c^{\prime}\right)$,

$$
\sum c_{(0)}^{\prime} c_{(0)} \otimes c_{(1)}^{\prime} c_{(1)} \otimes c_{(2)}=\sum c_{(0)} \otimes c_{(1)} \otimes c_{(2)}
$$

and it follows that

$$
\sum c_{(0)}^{\prime} c_{(0)} \otimes c_{(1)}^{\prime} c_{(1)} S\left(c_{(2)}\right)=\sum c_{(0)} \otimes c_{(1)} S\left(c_{(2)}\right)
$$

The above equality is nothing but $\chi(c)=c \otimes 1$. Now for any $a \in A$, we have

$$
\sum a_{(0)}\left(a_{(1)} \cdot c\right)=a c=c a
$$

and

$$
\sum c_{(0)}\left(c_{(1)} \cdot a\right)=c a=a c
$$


Thus $c$ is in both $A^{A}$ and ${ }^{A} A$. Now if $A$ is left or right strongly $H$-Azumaya then $c \in k$, and hence $M=c A=A$.

Lemma 2.21. If $A$ is a left or right strongly $H$-Azumaya algebra, then for any maximal $H$-ideal $M$ of $A$ there exists a maximal ideal $I$ of $k$ such that $M=I A$ and $M \cap k=I$.

Proof. Suppose that $A$ is left strongly $H$-Azumaya. Set $I=k \cap M$. Then $A / M$ is an $H$-simple left strongly $H$-Azumaya algebra over $k / I$ by Proposition 2.15 . Thus $k / I$ is a field. But $A / I A$ is also a left strongly $H$-Azumaya algebra over $k / I$ since $(A / I A)^{A / I A}=(A / I A)^{A}=k / I$. Therefore $A / I A$ is $H$-simple, and hence $I A=M$.

Let $A$ be a Yetter-Drinfel'd $H$-module algebra, and $B=A^{\# e}$ (resp. $B={ }^{\# e} A$ ). Denote by $\operatorname{Hom}_{B}^{H}(A, B)$ the abelian group of all morphisms in ${ }_{A \# e} \mathcal{Q}^{H}$ (resp. in $\mathcal{Q}_{\# \text { e }}^{H}$ ) from $A$ to $B$. Then the image $\operatorname{tr}_{B}(A)$ of the canonical morphism

$$
\operatorname{Hom}_{B}^{H}(A, B) \otimes A \longrightarrow B
$$

is called the $H$-trace of $A$. It is clear that $\operatorname{tr}_{B}(A)$ is an ideal of $A$. We have the following result:

Lemma 2.22. Let $A$ be strongly $H$-separable, and e a separability idempotent of $A^{\# e}$. Then $\operatorname{tr}_{B}^{H}(A)=B e B$.

If $A$ is strongly $H$-Azumaya, then $\operatorname{tr}_{B}(A)=B$.

Proof. We will prove the lemma in the case where $B=A^{\# e}$. The case $B={ }^{\#} A$ may be handled in a similar way. For $f \in \operatorname{Hom}_{B}^{H}(A, B), x \in A$, we have $f(x)=$ $(x \# \overline{1}) f(1)$, where $f(1)$ is obviously in ${ }^{A} B$. Thus $f(1)=e \rightarrow u=e u$ for some $u \in B$. It follows that $f(A) \subset B e B$. Since $e$ is clearly in $\operatorname{tr}_{B}(A)$ and $\operatorname{tr}_{B}(A)$ is an $H$-ideal of $B$, the first part is proved. Now the second part of the lemma follows from Lemma 2.21 and the fact that $\operatorname{tr}_{B}(A)$ is an $H$-ideal of $B$ and $B$ is also strongly $H$-Azumaya by Proposition 2.16 .

Theorem 2.23. Let $A$ be a Yetter-Drinfel'd $H$-module algebra. If $A$ is a strong $H$-Azumaya algebra, then $A$ is $H$-Azumaya.

Proof. In view of the results obtained above, the argument is identical to the one in the proof of the classical case; we refer to $[9,2.8]$.

In general, $H$-Azumaya algebras are not strongly $H$-Azumaya since an $H$ Azumaya algebra need not be a separable algebra. An example may be found in $[6,5.2]$. Now the following natural question arises: when is an $H$-Azumaya algebra strongly $H$-Azumaya?

Proposition 2.24. Let $A$ be an $H$-Azumaya algebra. Then the following assertions are equivalent:

1. A is strongly $H$-Azumaya algebra;

2. A has an $H$-trace map;

3. $k$ is an $H$-direct summand of $A$ (by an $H$-direct summand, we mean a direct summand as a Yetter-Drinfel'd H-module). 
Proof. 1) $\Rightarrow 3$ ) has been shown in 2.18. The equivalence of 2) and 3) is obvious. So let us show that 2) $\Rightarrow 1$ ). Let $t: A \longrightarrow k$ be an $H$-trace map of $A$. Since $A$ is $H$-Azumaya we have the isomorphism $A \# \bar{A} \cong \operatorname{End}(A)$, and we may view $t$ as an element in $A \# \bar{A}$. In fact $t$ is in $(A \# \bar{A})_{0}$ since $t$ is $H$-linear and $H$-colinear. Let us show that $t$ is an $H$-separability idempotent for $A^{\# e}$. Now $\pi_{A}(t)=t(1)=1$, and for any $a, x \in A$,

$$
(a \# 1) t(x)=a t(x)=(1 \# \bar{a}) t(x)
$$

because $t(x) \in k$. It follows from the above equalities that we have $(a \# 1) t=(1 \# \bar{a}) t$ for any $a \in A$. Therefore, $A$ is strongly $H$-separable.

Corollary 2.25. Let $A, B$ be $H$-Azumaya algebras. If $A \# B$ is strongly $H$-Azumaya, so are $A$ and $B$.

Proof. Since $A \# B$ is strongly $H$-Azumaya, $A \# B$ has an $H$-trace map $t$. Now the restriction $\left.t\right|_{A}(a)=t(a \# 1)$ is clearly an $H$-trace map of $A$; hence $A$ is strongly $H$-Azumaya. In a similar way, so is $B$.

The above corollary tells us that even the trivial $H$-Azumaya algebra $\operatorname{End}_{k}(M)$, $M$ a faithfully projective Yetter-Drinfel'd $H$-module, may be not strong. For example, if $A$ is non-strongly $H$-Azumaya, then $\bar{A}$ is non-strongly $H$-Azumaya by 2.16 , and hence $\operatorname{End}(A) \cong A \# \bar{A}$ is non-strongly $H$-Azumaya. So strongly $H$-Azumaya algebras may be Brauer equivalent to non-strongly $H$-Azumaya algebras. We have the following:

Theorem 2.26. The subset $\mathrm{BQS}(k, H)$ of classes of $H$-Azumaya algebras represented by a strongly $H$-Azumaya algebra is a subgroup of $\mathrm{BQ}(k, H)$.

Proof. This follows immediately from Proposition 2.16.

Now the question arises: when is $\mathrm{BQS}(k, H)$ equal to $\mathrm{BQ}(k, H)$ ? We know that if $A$ is an $H$-Azumaya algebra then $A$ is a projective $A^{\# e}$-module. If this projectivity can be lifted to the projectivity in ${ }_{A \# e} \mathcal{Q}^{H}$, then $A$ is strongly $H$-separable. In case $H$ is a Frobenius Hopf algebra or, more generally, a finitely generated projective Hopf algebra, the projectivity of $A$ in ${ }_{A^{\# e}} \mathcal{Q}^{H}$ is equivalent to $A$ being a projective $A^{\# e} \# \mathrm{D}(H)$-module. A sufficient condition ensuring this lifting property of the projectivity is that $\mathrm{D}(H)$ is 'semisimple-like' (that is, there exists a (left) integral $x \in H$ such that $\varepsilon(x)$ is a unit in $k$ ).

Proposition 2.27. If $H$ is a semisimple-like and cosemisimple-like Hopf algebra, then $\mathrm{BQS}(k, H)=\mathrm{BQ}(k, H)$.

Proof. By [22, Prop.8], $\mathrm{D}(H)$ is semisimple-like if and only if $H$ and $H^{*}$ are semisimple-like.

We remark that for a quasitriangular or coquasitriangular Hopf algebra $H$, the $H$-central and strongly $H$-separable or strongly $H$-Azumaya algebras are special cases of those above. For example, if $H$ is a semisimple-like quasitriangular Hopf algebra, then $\operatorname{BMS}(k, H)=\mathrm{BM}(k, H)$. If $G$ a finite abelian group and $H=k G$ with a bilinear map $\phi: G \times G \longrightarrow k$, then $H$ is a cosemisimple-like coquasitriangular Hopf algebra. Thus all graded $(H-)$ Azumaya algebras are strongly $H$-Azumaya, cf. $[9,10]$. 


\section{The SPlit Part of $\mathrm{BQ}(k, H)$}

3.1. Picard groups of Hopf algebras. Let $H$ be a Hopf algebra, and denote by $\mathrm{G}(H)$ the group of all grouplike elements in $H$. Write $\operatorname{Alg}(H, k)$ for the group of all algebra maps in the convolution algebra $\operatorname{Hom}(H, k)$. Consider the set

$$
\mathrm{E}(H)=\{g \bowtie \lambda \in \mathrm{G}(H) \times \operatorname{Alg}(H, k) \mid g(\lambda \rightarrow h)=(h-\lambda) g, \forall h \in H\},
$$

where $\lambda \rightarrow h=\sum h_{(1)} \lambda\left(h_{(2)}\right)$, and $h-\lambda=\sum \lambda\left(h_{(1)}\right) h_{(2)}$.

Lemma 3.1. $\mathrm{E}(H)$ is a group with multiplication given by

$$
(g \bowtie \lambda)\left(g^{\prime} \bowtie \lambda^{\prime}\right)=g^{\prime} g \bowtie \lambda \lambda^{\prime}
$$

Proof. $\mathrm{E}(H)$ is closed with respect to the above multiplication. Indeed, for $h \in H$,

$$
\begin{aligned}
g^{\prime} g\left(\lambda \lambda^{\prime}-h\right) & =\sum g^{\prime} g h_{(1)} \lambda\left(h_{(2)}\right) \lambda^{\prime}\left(h_{(3)}\right) \\
& =\sum g^{\prime} \lambda^{\prime}\left(h_{(3)}\right) h_{(2)} \lambda\left(h_{(1)}\right) g \\
& =\sum h_{(3)} \lambda^{\prime}\left(h_{(2)}\right) \lambda\left(h_{(1)}\right) g^{\prime} g \\
& =\sum\left(h-\lambda \lambda^{\prime}\right) g^{\prime} g
\end{aligned}
$$

For $g \bowtie \lambda \in \mathrm{E}(H)$ and $h \in H$, we have

$$
g(\lambda \rightarrow S(h))=(S(h)-\lambda) g
$$

That is, $\sum g \lambda^{-1}\left(h_{(1)}\right) S\left(h_{(2)}\right)=\sum \lambda^{-1}\left(h_{(2)}\right) S\left(h_{(1)}\right) g$. Now let $S^{-1}$ act on both sides of the above equality, to obtain

$$
\sum h_{(2)} \lambda^{-1}\left(h_{(1)}\right) g^{-1}=\sum g^{-1} h_{(1)} \lambda^{-1}\left(h_{(2)}\right)
$$

or

$$
\left(h \leftarrow \lambda^{-1}\right) g^{-1}=g^{-1}\left(\lambda^{-1} \rightarrow h\right)
$$

It follows that $g^{-1} \bowtie \lambda^{-1} \in \mathrm{E}(H)$. Finally, $1 \bowtie \varepsilon$ is the unit of the group $\mathrm{E}(H)$.

Definition 3.2. A Yetter-Drinfel'd $H$-module $I$ is called invertible if there exists a Yetter-Drinfel'd $H$-module $I^{\prime}$ such that $I \tilde{\otimes} I^{\prime} \simeq k$ as Yetter-Drinfel'd $H$-modules. The Yetter-Drinfel'd $H$-module isomorphism classes of invertible Yetter-Drinfel'd $H$-modules form a group under the tensor product $\tilde{\otimes}$, denoted by $\operatorname{PQ}(k, H)$, and is called the Picard group of the Hopf algebra $H$.

Similarly, for a quasitriangular Hopf algebra, the group of invertible $H$-modules is denoted by $\operatorname{PM}(k, H, R)$, and for a coquasitriangular Hopf algebra, the group of invertible $H$-comodules is denoted by $\mathrm{PC}(k, H, \mathcal{R})$

Proposition 3.3. Let $H$ be a Hopf algebra.

1. $\mathrm{PQ}(k, H) \simeq \operatorname{Pic}(k) \times \mathrm{E}(H)$;

2. If $H$ is a quasitriangular Hopf algebra, then $\operatorname{PM}(k, H) \simeq \operatorname{Pic}(k) \times \operatorname{Alg}(H, k)$;

3. If $H$ is a coquasitriangular Hopf algebra, then $\mathrm{PC}(k, H) \simeq \operatorname{Pic}(k) \times \mathrm{G}(H)$.

Proof. We will prove 1) and leave 2) and 3) to the reader. Define a map

$$
\xi: \operatorname{Pic}(k) \times \mathrm{E}(H) \longrightarrow P Q(k, H)
$$

by

$$
\xi([I], g \bowtie \lambda)=[I(g \bowtie \lambda)]
$$


where $I(g \bowtie \lambda)$ is equal to $I$ as a $k$-module but with $H$-structure given by

$$
h \cdot x=\lambda(h) x \text { and } \chi(x)=x \otimes g
$$

for $h \in H . \xi$ is well defined since

$$
\begin{aligned}
\chi(h \cdot x) & =\lambda(h) x \otimes g \\
& =x \otimes \lambda(h) g \\
& =\sum x \otimes g h_{(2)} \lambda\left(h_{(3)}\right) S^{-1}\left(h_{(1)}\right) \\
& =\sum \lambda\left(h_{(2)}\right) x \otimes h_{(3)} g S^{-1}\left(h_{(1)}\right) \\
& =\sum h_{(2)} \cdot x \otimes h_{(3)} g S^{-1}\left(h_{(1)}\right)
\end{aligned}
$$

It is clear that $I^{\prime}\left(g^{-1} \bowtie \lambda^{-1}\right)$ is an inverse for $I(g \bowtie \lambda)$. Conversely, for $I \in$ $P Q(k, H)$, consider $I^{\prime}$ such that $I \tilde{\otimes} I^{\prime} \simeq k$ in the category $\mathcal{Q}^{H}$. Forget the $H$ structure on $I^{\prime}$ and denote the resulting $k$-module by $\underline{I}^{\prime}$. Then the Yetter-Drinfel'd $H$-module $J=I \otimes \underline{I}^{\prime}$ with $H$-structure stemming from $I$ is isomorphic to $k$ as a $k$-module. We may therefore write $J=k m$, for some $m \in J$, and it follows that $\chi(m)=m \otimes g$ for some $g \in H$. It is easy to see that $g \in G(H)$. On the other hand, for any $h \in H, h \cdot m=k_{h} m \in k m$ for some $k_{h} \in k$. Define $\lambda: H \longrightarrow k$ by $\lambda(h)=k_{h}$. The $H$-module structure on $J$ makes $\lambda$ into an algebra map, so $\lambda \in \operatorname{Alg}(H, k)$. Now we claim that $\sigma=g \bowtie \lambda \in \mathrm{E}(H)$. Indeed, for all $h \in H$, we have that

$$
\chi(h \cdot m)=\sum h_{(2)} \cdot m \otimes h_{(3)} g S^{-1}\left(h_{(1)}\right)=\sum m \otimes \lambda\left(h_{(2)}\right) h_{(3)} g S^{-1}\left(h_{(1)}\right)
$$

Now $\chi(h \cdot m)=\chi(\lambda(h) m)=m \otimes \lambda(h) g$, and it follows that

$$
\lambda(h) g=\sum \lambda\left(h_{(2)}\right) h_{(3)} g S^{-1}\left(h_{(1)}\right) .
$$

Therefore,

$$
\begin{aligned}
g(\lambda \rightarrow h) & =\sum g \lambda\left(h_{(2)}\right) h_{(1)}=\sum \lambda\left(h_{(3)}\right) h_{(4)} g S^{-1}\left(h_{(2)}\right) h_{(1)} \\
& =\sum \lambda\left(h_{(1)}\right) h_{(2)} g=(h \leftarrow \lambda) g
\end{aligned}
$$

The element $\sigma$ is uniquely determined by $I$, so we have a well-defined map

$$
\eta: \mathrm{PQ}(k, H) \longrightarrow \mathrm{Pic}(k) \times \mathrm{E}(H),[I] \mapsto[\underline{I}] \times \sigma_{I}
$$

It is not hard to see that $\xi$ and $\eta$ are group maps and are each others inverses.

Remark 3.4. Note that $\mathrm{PQ}(k, H)$ is an abelian group, since the tensor product $\tilde{\otimes}$ is naturally commutative. It follows that $\mathrm{E}(H)$ is an abelian group. In particular, if $H$ is a quasitriangular (resp. a coquasitriangular) Hopf algebra, then $\operatorname{Alg}(H, k)$ (resp. $G(H)$ ) is abelian. As an example, if $H$ is faithfully projective, then $G(D(H))$, the grouplike elements of the Drinfel'd double, form an abelian group. Moreover, if the elements $\sigma=g \bowtie \lambda$ and $\sigma^{\prime}=g^{\prime} \bowtie \lambda^{\prime}$ are in $\mathrm{E}(H)$, then $g g^{\prime}=g^{\prime} g$ and $\lambda \lambda^{\prime}=\lambda^{\prime} \lambda$.

3.2. The generalized Rosenberg-Zelinsky exact sequence. Let $A$ be a Yetter-Drinfel'd $H$-module algebra. Denote by $H$ - $\operatorname{Aut}(A)$ the group of all YetterDrinfel'd $H$-module algebra automorphisms of $A$. We call $f \in H$ - Aut $(A) H$-INNER if there exists an invertible element $x \in A_{0}$ such that $f(a)=x a x^{-1}$, for all $a \in A$.

The subgroup of $H$ - $\operatorname{Aut}(A)$ consisting of $H$-INNER automorphisms of $A$ will be denoted by $H$ - $\operatorname{INN}(A) . f \in H$ - $\operatorname{Aut}(A)$ is called $H$-inner if there exist an invertible element $x \in A$ and a grouplike element $g \in G(H)$ such that $f(a)=x(g \cdot a) x^{-1}$ for 
all $a \in A$. We have the following generalization of the Rosenberg-Zelinsky exact sequence:

Proposition 3.5. For an $H$-Azumaya algebra $A$, we have the exact sequence

$$
1 \longrightarrow H-\operatorname{INN}(A) \longrightarrow H-\operatorname{Aut}(A) \stackrel{\Phi}{\longrightarrow} P Q(k, H)
$$

where

$$
\Phi(f)=I_{f}=\left\{x \in A \mid \sum x_{(0)}\left(x_{(1)} \cdot a\right)=f(a) x, \forall a \in A\right\}
$$

Proof. Observe that $A$ is an object of the monoidal category ${ }_{A} \mathcal{Q}_{A}^{H}$ of $(H, A$ - $A)$ bimodules. For $\alpha, \beta \in H$ - $\operatorname{Aut}(A)$, let ${ }_{\alpha} A_{\beta}$ be the object of ${ }_{A} \mathcal{Q}_{A}^{H}$ which is equal to $A$ as a Yetter-Drinfel'd $H$-module algebra but with $A$-bimodule structure given by

$$
a \rightarrow x=\alpha(a) x, \quad x \leftarrow a=x \beta(a)
$$

Let $\alpha, \beta$ and $\gamma \in H$ - $\operatorname{Aut}(A)$. It is easy to check that we have the following isomorphisms in ${ }_{A} \mathcal{Q}_{A}^{H}$ (cf. [13, Ch.IV]):

1. ${ }_{\alpha} A_{\beta} \simeq{ }_{\gamma \alpha} A_{\gamma \beta}($ via $x \mapsto \gamma(x))$;

2. ${ }_{\alpha} A_{\beta} \tilde{\otimes}_{A}{ }_{\gamma} A_{\beta} \simeq{ }_{\gamma \alpha} A_{\beta}$ and ${ }_{\alpha} A_{\beta} \tilde{\otimes}_{A \alpha} A_{\gamma} \simeq{ }_{\alpha} A_{\beta \gamma}$;

3. ${ }_{\alpha} A_{1} \simeq{ }_{1} A_{1}$ if and only if $\alpha \in H-\operatorname{INN}(A)$.

Take $\alpha \in H$-Aut $(A)$. It follows from Proposition 2.7 that

$$
{ }_{\alpha} A_{1} \simeq A \tilde{\otimes}\left({ }_{\alpha} A_{1}\right)^{A}
$$

Write $I_{\alpha}$ for $\left({ }_{\alpha} A_{1}\right)^{A}$. Then $I_{\alpha}$ is an invertible Yetter-Drinfel'd $H$-module with inverse $I_{\alpha^{-1}}$ by formula 2 ) above. Observe that

$$
I_{\alpha}=\Phi(\alpha)=\left\{x \in{ }_{\alpha} A_{1} \mid \alpha(a) x=\sum x_{(0)}\left(x_{(1)} \cdot a\right), \forall a \in A\right\}
$$

as required. Now 2) and Proposition 2.7 imply that $\Phi$ is a homomorphism, since

$$
I_{\alpha \beta} \simeq\left({ }_{\alpha \beta} A_{1}\right)^{A} \simeq\left({ }_{\beta} A_{1} \tilde{\otimes}_{A}{ }_{\alpha} A_{1}\right)^{A} \simeq\left({ }_{\beta} A_{1}\right)^{A} \tilde{\otimes}\left({ }_{\alpha} A_{1}\right)^{A} \simeq I_{\beta} \tilde{\otimes} I_{\alpha} \simeq I_{\alpha} \tilde{\otimes} I_{\beta}
$$

From 3 ) it now follows that the sequence

$$
1 \longrightarrow H-\operatorname{INN}(A) \longrightarrow H-\operatorname{Aut}(A) \stackrel{\Phi}{\longrightarrow} P Q(k, H)
$$

is exact.

Corollary 3.6 (Generalized Skolem-Noether Theorem). If $\operatorname{Pic}(k)=1$ and $A$ is $H$-Azumaya, then each $\alpha \in H$-Aut $(A)$ is $H$-inner.

Proof. Since $\left[I_{\alpha}\right]=1$ in $\operatorname{Pic}(k), I_{\alpha}=k x$ for some invertible element $x \in A$. Let $\left[I_{\alpha}\right]=([k], g \bowtie \lambda)$ in $\mathrm{PQ}(k, H) \simeq \operatorname{Pic}(k) \times \mathrm{E}(H)$. Then $h \cdot x=\lambda(h) x$ for all $h \in H$, and $\chi(x)=x \otimes g$.

Now let $\alpha \in \operatorname{Aut}(A)$. By (22) we have

$$
\alpha(a) x=\sum x_{(0)}\left(x_{(1)} \cdot a\right)=x(g \cdot a) .
$$

Thus $\alpha(a)=x(g \cdot a) x^{-1}$ for all $a \in A$.

Remark 3.7. If $H$ is a coquasitriangular Hopf algebra, then there are comodule versions of (3.5) and (3.6) for an $H$-Azumaya $H^{o p}$-comodule algebra $A$. Similarly, for a quasitriangular Hopf algebra $(H, R)$ and an $H$-Azumaya $H$-module algebra $A$, we have a module version of (3.5) and (3.6). The condition that an automorphism is $H$-inner is then as follows: $f \in H$-Aut $(A)$ is $H$-inner if there exist $\lambda \in \operatorname{Alg}(H, k)$ and an invertible element $x \in A$ such that for all $a \in A, f(a)=x(u \cdot a) x^{-1}$, where $u=\sum \lambda\left(R^{(2)}\right) R^{(1)}$. 
3.3. The map $\beta$. Recall that the Brauer group of Yetter-Drinfel'd $H$-module algebras is functorial in $k$ : if $\ell$ is a commutative $k$-algebra, then we have a natural map $\mathrm{BQ}(k, H) \longrightarrow \mathrm{BQ}(\ell, \ell \otimes H)$. As usual, we will denote

$$
\mathrm{BQ}(\ell / k, H)=\operatorname{Ker}(\mathrm{BQ}(k, H) \longrightarrow \mathrm{BQ}(\ell, \ell \otimes H))
$$

and

$$
\mathrm{BQ}^{s}(k, H)=\bigcup_{\ell} \mathrm{BQ}(\ell / k, H) \subset \mathrm{BQ}(k, H)
$$

where the union is taken over all faithfully flat $k$-algebras $\ell$. In the case where $H$ is commutative, cocommutative and faithfully projective, an exact sequence connecting $\mathrm{BQ}^{s}(k, H)$ and $\mathrm{BQ}(k, H)$ was given by the first author in $[5,6]$. In this subsection we will generalize this result and derive a complex involving $\mathrm{BQ}(k, H)$, the split part $\mathrm{BQ}^{s}(k, H)$ and the automorphism group of $\mathrm{E}(H)$ :

$$
1 \longrightarrow \mathrm{BQ}^{s}(k, H) \longrightarrow \mathrm{BQ}(k, H) \stackrel{\beta}{\longrightarrow} \mathrm{O}(\mathrm{E}(H)) \longrightarrow 1
$$

Let $\mathrm{E}(H)$ be the abelian group described in (20). Consider an $H$-Azumaya algebra $A$. We define a map

$$
\mathrm{E}(H) \stackrel{\rho}{\longrightarrow} \operatorname{End}_{k}(A)
$$

by

$$
\rho(g \bowtie \lambda)(a)=\sum\left(g \cdot a_{(0)}\right) \lambda\left(a_{(1)}\right)
$$

for $a \in A, \sigma=g \bowtie \lambda$. The image of $\rho$ is in $H$ - $\operatorname{Aut}(A)$. In fact, for $h \in H$, we have

$$
\begin{aligned}
\rho(\sigma)(h \cdot a) & =\sum\left(g h_{(2)} \cdot a_{(0)}\right) \lambda\left(h_{(3)} a_{(1)} S^{-1}\left(h_{(1)}\right)\right) \\
& =\sum g\left(\lambda-h_{(2)}\right) \cdot a_{(0)} \lambda\left(a_{(1)}\right) \lambda^{-1}\left(h_{(1)}\right) \\
& =\sum\left(h_{(2)}-\lambda\right) g \cdot a_{(0)} \lambda\left(a_{(1)}\right) \lambda^{-1}\left(h_{(1)}\right) \\
& =\sum h \cdot\left(g \cdot a_{(0)}\right) \lambda\left(a_{(1)}\right) \\
& =h \cdot(\rho(\sigma)(a))
\end{aligned}
$$

and

$$
\begin{aligned}
\chi(\rho(\sigma)(a)) & =\sum \chi\left(g \cdot a_{(0)}\right) \lambda\left(a_{(1)}\right) \\
& =\sum g \cdot a_{(0)} \otimes g a_{(1)} g^{-1} \lambda\left(a_{(2)}\right) \\
& =\sum g \cdot a_{(0)} \otimes g\left(\lambda-a_{(1)}\right) g^{-1} \\
& =\sum g \cdot a_{(0)} \otimes a_{(1)}-\lambda \\
& =\rho(\sigma)\left(a_{(0)}\right) \otimes a_{(1)}
\end{aligned}
$$

Moreover, the inverse of $\rho(\sigma)$ in $H$ - $\operatorname{Aut}(A)$ is $\rho\left(\sigma^{-1}\right)$. Now we define $\alpha_{A}$ to be the following composition:

$$
\alpha_{A}: \mathrm{E}(H) \stackrel{\rho}{\longrightarrow} H-\operatorname{Aut}(A) \stackrel{\Phi}{\longrightarrow} \mathrm{PQ}(k, H) \stackrel{(3.3)}{\longrightarrow} \mathrm{E}(H)
$$

$\rho$ is a homomorphism in view of Remark 3.4. It follows that $\alpha_{A}$ is a homomorphism. The map $\beta_{A}: \mathrm{E}(H) \longrightarrow \mathrm{E}(H)$ is defined by

$$
\beta_{A}(\sigma)=\sigma \alpha_{A}(\sigma)^{-1}
$$


Given $\sigma=g \bowtie \lambda \in \mathrm{E}(H)$, we have

$$
\begin{aligned}
\Phi(\rho(\sigma)) & =I_{\sigma}=\left\{x \in A \mid \sigma(a) x=\sum x_{(0)}\left(x_{(1)} \cdot a\right), \forall a \in A\right\} \\
& =\left\{x \in A \mid \sum\left(g \cdot a_{(0)}\right) x \lambda\left(a_{(1)}\right)=\sum x_{(0)}\left(x_{(1)} \cdot a\right), \forall a \in A\right\}
\end{aligned}
$$

Lemma 3.8. Let $A, B$ be $H$-Azumaya algebras. Then $\beta_{A \# B}=\beta_{A} \circ \beta_{B}$.

Proof. First we show $\left(\alpha_{A} \circ \beta_{B}\right) * \alpha_{B}=\alpha_{A \# B}$. Take $\sigma=g \bowtie \lambda \in \mathrm{E}(H)$ and let $\alpha_{B}(\sigma)=p \bowtie \theta$. Set $\left(\alpha_{A} \circ \beta_{B}\right)(\sigma)=\alpha_{A}\left(\beta_{B}(\sigma)\right)=q \bowtie \eta$. For $u \in \Phi_{A}\left(\rho\left(\beta_{B}(\sigma)\right)\right)$ and $v \in \Phi_{B}(\rho(\sigma))$, we have

$$
h \cdot u=\eta(h) u, \chi(u)=u \otimes q \text { and } h \cdot v=\theta(h) v, \chi(v)=v \otimes p .
$$

The following computation implies that $u \# v$ is in $\Phi_{A \# B}(\rho(\sigma))$. For $a \# b \in A \# B$, write $\rho(\sigma)(x)=\sigma(x)$,

$$
\begin{aligned}
{[\rho(\sigma)(a \# b)](u \# v) } & =(\sigma(a) \# \sigma(b))(u \# v) \\
& =\sum \sigma(a) u_{(0)} \#\left(u_{(1)} \cdot \sigma(b)\right) v \\
& =\sigma(a) u \#(q \cdot \sigma(b)) v \\
& =\beta_{B}(\sigma)\left(\alpha_{B}(\sigma)(a)\right) u \# \sigma(q \cdot b) v \\
& =u\left(q \cdot \alpha_{B}(\sigma)(a)\right) \# v(p q \cdot b) \\
& =u\left(\alpha_{B}(\sigma)(q \cdot a) \# v(p q \cdot b)\right. \\
& =\sum u\left(p q \theta\left(a_{(1)}\right) \cdot a_{(0)}\right) \# v(p q \cdot b) \\
& =\sum u\left(p q \cdot a_{(0)}\right) \#\left(p q a_{(1)} q^{-1} p^{-1} \cdot v\right)(p q \cdot b) \\
& =(u \# v)(p q \cdot(a \# b)) \\
& =\sum(u \# v)_{(0)}(u \# v)_{(1)} \cdot(a \# b)
\end{aligned}
$$

It follows that

$$
\alpha_{A \# B}(\sigma)=p q \bowtie \eta \theta=\left(\alpha_{A} \circ \beta_{B}\right) * \alpha_{B}(\sigma)
$$

Now

$$
\begin{aligned}
\beta_{A \# B}(\sigma) & =\sigma \alpha_{A \# B}(\sigma)^{-1} \\
& =\sigma\left[\left(\alpha_{A} \circ \beta_{B}\right)(\sigma) \alpha_{B}(\sigma)\right]^{-1} \\
& =\sigma \alpha_{B}(\sigma)^{-1} \alpha_{A}\left(\beta_{B}(\sigma)\right)^{-1} \\
& =\beta_{B}(\sigma) \alpha_{A}\left(\beta_{B}(\sigma)\right)^{-1} \\
& =\beta_{A} \circ \beta_{B}(\sigma)
\end{aligned}
$$

and this finishes the proof.

Lemma 3.9. If $P \in \mathcal{Q}^{H}$ is faithfully projective, and $A=\operatorname{End}(P)$, then $\beta_{A}=$ $I_{\mathrm{E}(H)}$.

Proof. Given $\sigma=g \bowtie \lambda \in \mathrm{E}(H)$, we define $\psi_{\sigma}: P \longrightarrow P$ by $\psi_{\sigma}(x)=\sigma(x)=$ $\sum g \cdot x_{(0)} \lambda\left(x_{(1)}\right)$. Then $\psi_{\sigma} \in H$-Aut $(P)$, since $\rho(\sigma) \in H$-Aut $(A)$. One may easily see that $\psi_{\sigma}$ is in $\operatorname{End}(P)_{0}$, since $\psi_{\sigma}$ is $H$-linear and $H$-colinear. Furthermore $\psi_{\sigma}$ is 
in $\Phi_{A}(\rho(\sigma))$, and hence $\alpha_{A}(\sigma)=1 \otimes \varepsilon$. Indeed, for $f \in A$,

$$
\begin{aligned}
\rho(\sigma)(f) \circ \psi_{\sigma}(x) & =\rho(\sigma)(f)(\sigma(x)) \\
& =\sum g \cdot f_{(0)}\left(g^{-1} g \cdot x_{(0)}\right) \lambda\left(x_{(1)} f_{(1)}\right) \\
& =\sum g \cdot f\left(x_{(0)}\right)_{(0)} \lambda\left(x_{(2)} S^{-1}\left(x_{(1)}\right) f\left(x_{(0)}\right)_{(1)}\right) \\
& =\sigma \cdot f(x)=\psi_{\sigma} \circ f(x)
\end{aligned}
$$

Therefore, $\beta_{A}=I_{\mathrm{E}(H)}$.

Lemma 3.10. $\beta_{A} \in \operatorname{Aut}(\mathrm{E}(H))$.

Proof. Since $\alpha_{A}$ is a group homomorphism and $\mathrm{E}(H)$ is abelian, it follows that $\beta_{A}$ is in $\operatorname{End}(\mathrm{E}(H))$. By (4.8) and (4.9), $\beta_{\bar{A}}$ is an inverse for $\beta_{A}$, and therefore $\beta_{A} \in \operatorname{Aut}(\mathrm{E}(H))$.

The above arguments may be summarized as follows:

Proposition 3.11. We have a group homomorphism

$$
\beta: \mathrm{BQ}(k, H) \longrightarrow \operatorname{Aut}(\mathrm{E}(H))
$$

defined by $\beta([A])=\beta_{A}$.

Define a subgroup $\mathrm{O}(\mathrm{E}(H))$ of $\operatorname{Aut}(\mathrm{E}(H))$ as follows:

$$
\mathrm{O}(\mathrm{E}(H))=\{f \in \operatorname{Aut}(\mathrm{E}(H)) \mid \omega \circ f=\omega\},
$$

where $\omega: \mathrm{E}(H) \longrightarrow \mathbb{G}_{m}(k)$ is given by $\omega(g \bowtie \lambda)=\langle\lambda, g\rangle$.

Lemma 3.12. $\operatorname{Im}(\beta) \subset \mathrm{O}(\mathrm{E}(H))$.

Proof. Let $f \in \operatorname{Im}(\beta)$. That is, $f=\beta_{A}$ for some $H$-Azumaya algebra $A$. Take $\sigma=g \bowtie \lambda \in \mathrm{E}(H)$. Set $\alpha_{A}(\sigma)=p \bowtie \theta$. Suppose $u \in \Phi_{A}\left(\rho\left(\sigma^{-1}\right)\right)$. Then $\chi(u)=u \otimes p^{-1}$, and $h \cdot u=\theta^{-1}(h) u=\left\langle\theta^{-1}, h\right\rangle u$ for all $h \in H$.

Since $u$ is in $\Phi_{A}\left(\rho\left(\sigma^{-1}\right)\right)$, we have

$$
\rho\left(\sigma^{-1}\right)(u) u=u\left(p^{-1} \cdot u\right) .
$$

That is,

$$
\left\langle\lambda^{-1}, p^{-1}\right\rangle\left\langle\theta^{-1}, g^{-1}\right\rangle u^{2}=u^{2}\left\langle\theta^{-1}, p^{-1}\right\rangle .
$$

If $k$ is local, then by (3.6) $\rho\left(\sigma^{-1}\right)$ is $H$-inner. We may choose $u$ to be an invertible element in $A$, and then we obtain

$$
\left\langle\lambda^{-1}, p^{-1}\right\rangle\left\langle\theta^{-1}, g^{-1}\right\rangle=\left\langle\theta^{-1}, p^{-1}\right\rangle .
$$

Now a local-global argument implies that (25) holds for an arbitrary commutative ring $k$. Thus,

$$
\begin{aligned}
\omega \circ \beta_{A}(\sigma) & =\omega\left(\sigma \alpha_{A}(\sigma)^{-1}\right) \\
& =\omega\left(p^{-1} g \bowtie \lambda \theta^{-1}\right)=\left\langle\lambda \theta^{-1}, p^{-1} g\right\rangle \\
& =\left\langle\lambda, p^{-1}\right\rangle\langle\lambda, g\rangle\left\langle\theta^{-1}, p^{-1}\right\rangle\left\langle\theta^{-1}, g\right\rangle \\
& =\langle\lambda, g\rangle\left\langle\lambda, p^{-1}\right\rangle\left\langle\lambda^{-1}, p^{-1}\right\rangle\left\langle\theta^{-1}, g^{-1}\right\rangle\left\langle\theta^{-1}, g\right\rangle \\
& =\langle\lambda, g\rangle=\omega(\sigma) .
\end{aligned}
$$

Therefore $\beta_{A} \in \mathrm{O}(\mathrm{E}(H))$.

Lemma 3.13.

$$
\mathrm{BQ}^{s}(k, H) \subset \operatorname{Ker}(\beta)
$$


Proof. Suppose that $A$ is an $H$-Azumaya algebra such that $A \otimes K \cong \operatorname{End}_{K}(P)$ for some faithfully flat $k$-algebra $K$ and some $K$-faithfully projective Yetter-Drinfel'd $H$-module $P$. Then $A \otimes K$ is $H$-Azumaya over $K$ by Proposition 2.15 and $\beta_{A \otimes K}=$ $I_{\mathrm{E}(H \otimes K)}$ by Lemma 3.9. Since $K$ is faithfully flat over $k, \mathrm{E}(H)$ is a subgroup of the abelian group $\mathrm{E}(H \otimes K)$. Now

$$
\beta_{A \otimes K}: \mathrm{E}(H \otimes K) \longrightarrow \mathrm{E}(H \otimes K)
$$

restricted to $\mathrm{E}(H)$ is nothing else but $\beta_{A}$. Therefore $\beta_{A}=I_{\mathrm{E}(H)}$.

Proposition 3.14. Let $H$ be a Hopf algebra. We then have a complex

$$
1 \longrightarrow \mathrm{BQ}^{s}(k, H) \longrightarrow \mathrm{BQ}(k, H) \stackrel{\beta}{\longrightarrow} \mathrm{O}(\mathrm{E}(H))
$$

\section{Hopf AUTOMORPhism GROUPS}

In this section, $H$ will be a faithfully projective Hopf algebra over $k$. We will construct an anti-homomorphism from $\operatorname{Aut}_{\text {Hopf }}(H)$ to $\mathrm{BQ}(k, H)$, and we will show that its image determines the action of $\operatorname{Aut}_{\text {Hopf }}(H)$ on $\mathrm{BQ}(k, H)$.

We know that if $M$ is a faithfully projective Yetter-Drinfel'd $H$-module, then $\operatorname{End}_{k}(M)$ is an $H$-Azumaya Yetter-Drinfel'd $H$-module algebra. However, if $M$ is an $H$-bimodule, that is, a left $H$-module and a right $H$-comodule, but not a Yetter-Drinfel'd $H$-module, it may still happen that $\operatorname{End}_{k}(M)$ is a Yetter-Drinfel'd $H$-module algebra. Let us provide an example first.

Let $H$ be a faithfully projective Hopf algebra. Take a non-trivial Hopf algebra isomorphism $\alpha \in \operatorname{Aut}_{\text {Hopf }}(H)$ (for example, if the antipode $S$ of $H$ is not of order 2, $S^{2}$ is a non-trivial Hopf automorphism). We define an $H$-bimodule $H_{\alpha}$ as follows:

As a $k$-module $H_{\alpha}=H$; we give $H_{\alpha}$ the obvious $H$-comodule structure given by $\Delta$, and an $H$-module structure as follows :

$$
h \cdot x=\sum \alpha\left(h_{(2)}\right) x S^{-1}\left(h_{(1)}\right)
$$

for $h \in H, x \in H_{\alpha}$. Since $\alpha$ is nontrivial, $H_{\alpha}$ is not a Yetter-Drinfel'd $H$-module but an $H$-bimodule. Let $A_{\alpha}=\operatorname{End}\left(H_{\alpha}\right)$ with $H$-structures induced by the $H$-structures of $H_{\alpha}$, that is,

$$
\begin{aligned}
(h \cdot f)(x) & =\sum h_{(1)} f\left(S\left(h_{(2)}\right) \cdot x\right) \\
\chi(f)(x) & =\sum f\left(x_{(0)}\right)_{(0)} \otimes S^{-1}\left(x_{(1)}\right) f\left(x_{(0)}\right)_{(1)}
\end{aligned}
$$

for $f \in A_{\alpha}, x \in H_{\alpha}$.

Lemma 4.1. If $H$ is a faithfully projective Hopf algebra and $\alpha$ is a Hopf algebra automorphism of $H$, then $A_{\alpha}$ is a Yetter-Drinfel'd $H$-module algebra.

Proof. We identify $A_{\alpha}$ with $H_{\alpha} \otimes H_{\alpha}^{*}$, where the left action on $H_{\alpha}^{*}$ is given by

$$
(h \cdot p)(x)=\langle p, S(h) \cdot x\rangle
$$

and the right $H$-coaction by

$$
\chi(p)(x)=\sum\left\langle p, x_{(0)}\right\rangle \otimes S^{-1}\left(x_{(1)}\right)
$$


for $h \in H, p \in H_{\alpha}^{*}$ and $x \in H_{\alpha}$. Take $f=m \otimes m^{*} \in A_{\alpha}, x \in H_{\alpha}$; then we have

$$
\begin{aligned}
\chi & (h \cdot f)(x)=\sum(h \cdot f)\left(x_{(0)}\right)_{(0)} \otimes S^{-1}\left(x_{(1)}\right)(h \cdot f)\left(x_{(0)}\right)_{(1)} \\
& =\sum \alpha\left(h_{(3)}\right) m_{(0)} S^{-1}\left(h_{(2)}\right)\left\langle\left(h_{(5)} \cdot m^{*}\right)_{(0)}, x\right\rangle \otimes\left(h_{(5)} \cdot m^{*}\right)_{(1)} \alpha\left(h_{(4)}\right) m_{(1)} S^{-1}\left(h_{(1)}\right) \\
& =\sum \alpha\left(h_{(3)}\right) m_{(0)} S^{-1}\left(h_{(2)}\right)\left\langle h_{(5)} \cdot m^{*}, x_{(0)}\right\rangle \otimes S^{-1}\left(x_{(1)}\right) \alpha\left(h_{(4)}\right) m_{(1)} S^{-1}\left(h_{(1)}\right) \\
& =\sum h_{(2)} \cdot m_{(0)}\left\langle m^{*}, S\left(h_{(4)}\right) \cdot x_{(0)}\right\rangle \otimes S^{-1}\left(x_{(1)}\right) \alpha\left(h_{(3)}\right) m_{(1)} S^{-1}\left(h_{(1)}\right)
\end{aligned}
$$

On the other hand,

$$
\begin{aligned}
\sum & \left(h_{(2)} \cdot f_{(0)}\right)(x) \otimes h_{(3)} f_{(1)} S^{-1}\left(h_{(1)}\right) \\
= & \sum\left(h_{(2)} \cdot m_{(0)} \otimes h_{(3)} \cdot m_{(0)}^{*}\right)(x) \otimes h_{(4)} m_{(1)}^{*} m_{(1)} S^{-1}\left(h_{(1)}\right) \\
= & \sum\left(h_{(2)} \cdot m_{(0)}\right)\left\langle m_{(0)}^{*}, S\left(h_{(3)}\right) \cdot x\right\rangle \otimes h_{(4)} m_{(1)}^{*} m_{(1)} S^{-1}\left(h_{(1)}\right) \\
= & \sum\left(h_{(2)} \cdot m_{(0)}\right)\left\langle m^{*}, S\left(h_{(4)}\right) \cdot x_{(0)}\right\rangle \\
& \otimes h_{(6)} S^{-1}\left(\alpha\left(S\left(h_{(3)}\right)\right) x_{(1)} h_{(5)}\right) m_{(1)} S^{-1}\left(h_{(1)}\right) \\
= & \sum h_{(2)} \cdot m_{(0)}\left\langle m^{*}, S\left(h_{(4)} \cdot x_{(0)}\right\rangle \otimes S^{-1}\left(x_{(1)}\right) \alpha\left(h_{(3)}\right) m_{(1)} S^{-1}\left(h_{(1)}\right)\right.
\end{aligned}
$$

It follows that $A_{\alpha}$ is a Yetter-Drinfel'd $H$-module. Since the $H$-module and $H^{o p}$ comodule algebra structure are independent of the Yetter-Drinfel'd compatibility condition, $A_{\alpha}$ is a Yetter-Drinfel'd $H$-module algebra.

In the sequel, we will see that $A_{\alpha}$ is $H$-Azumaya. Now let $M, N$ be faithfully projective $H$-bimodules such that $\operatorname{End}_{k}(M), \operatorname{End}_{k}(N)$ are Yetter-Drinfel'd $H$-module algebras. For $h \in H$, let $\rho(h)$ be the map $M \longrightarrow M$ defined by $\rho(h)(m)=h \dot{m}$. It is clear that $\rho(h l)=\rho(h) \rho(l)$, for $h, l \in H$.

Proposition 4.2. With assumptions as above, $\operatorname{End}(M) \# \operatorname{End}(N) \cong \operatorname{End}(M \otimes N)$ as Yetter-Drinfel'd $H$-module algebras.

Proof. As shown in $[8,(4.3)]$, the map

$$
\phi: \operatorname{End}(M) \# \operatorname{End}(N) \longrightarrow \operatorname{End}(M \otimes N)
$$

given by $\phi(f \# g)(m \otimes n)=\sum f\left(m_{(0)}\right) \otimes\left(m_{(1)} \cdot g\right)(n)$ is an $H^{o p}$-comodule algebra isomorphism, but is not $H$-linear in our case. We will define another $H$-action on $M \otimes N$ such that the induced $H$-module structure on $\operatorname{End}_{k}(M \otimes N)$ makes the map $\phi$ into an $H$-module map, and this will finish our proof. We need the following lemma.

Lemma 4.3. Let $M$ be a $k$-module such that $\operatorname{End}_{k}(M)$ is an $H$-module algebra. If the $H$-action on $\operatorname{End}_{k}(M)$ is strongly inner, that is, there exists an algebra map $u: H \longrightarrow \operatorname{End}_{k}(M)$ such that $h \cdot f=\sum u\left(h_{(1)}\right) f u\left(S\left(h_{(2)}\right)\right)$, then there is an $H$-action on $M$ inducing the $H$-action on $\operatorname{End}_{k}(M)$.

Proof. The desired action of $H$ on $M$ is given by $h \cdot m=u(h)(m)$, and $M$ with this $H$-structure is an $H$-module.

We are now able to finish the proof of Proposition 4.2 : Define a map

$$
\eta: \operatorname{End}(M) \# \operatorname{End}(N) \longrightarrow \operatorname{End}(N) \otimes \operatorname{End}(M)
$$


as follows:

$$
\eta(f \# g)=\sum \rho\left(S\left(f_{(1)}\right)\right) g \otimes f_{(0)}
$$

$\eta$ is an algebra isomorphism. Indeed, for $f, f^{\prime} \in \operatorname{End}_{k}(M)$ and $g, g^{\prime} \in \operatorname{End}_{k}(N)$, we have

$$
\begin{aligned}
\eta\left((f \# g)\left(f^{\prime} \# g^{\prime}\right)\right) & =\sum \eta\left(f f_{(0)}^{\prime} \#\left(f_{(1)}^{\prime} \cdot g\right) g^{\prime}\right) \\
& =\sum \rho\left(S\left(f_{(1)}^{\prime} f_{(1)}\right)\right)\left(f_{(2)}^{\prime} \cdot g\right) g^{\prime} \otimes f_{(0)} f_{(0)}^{\prime} \\
& =\sum \rho\left(S\left(f_{(1)}\right) \rho\left(S\left(f_{(1)}^{\prime}\right)\right) \rho\left(f_{(2)}^{\prime}\right) g \rho\left(S\left(f_{(3)}^{\prime}\right)\right) g^{\prime} \otimes f_{(0)} f_{(0)}^{\prime}\right. \\
& =\eta(f \# g) \eta\left(f^{\prime} \# g^{\prime}\right)
\end{aligned}
$$

Identifying $\operatorname{End}_{k}(N) \otimes \operatorname{End}_{k}(M)$ with $\operatorname{End}_{k}(N \otimes M)$, we obtain an algebra isomorphism

$$
\eta: \operatorname{End}(M) \# \operatorname{End}(N) \longrightarrow \operatorname{End}(N \otimes M)
$$

Let us show that $\eta$ is an $H$-module algebra isomorphism. For $h \in H, f \in \operatorname{End}(M)$ and $g \in \operatorname{End}(N)$, we have

$$
\begin{aligned}
\sum & \eta\left(h_{(1)} \cdot f \# h_{(2)} \cdot g\right) \\
& =\sum \rho\left(S\left(h_{(3)} f_{(1)} S^{-1}\left(h_{(1)}\right)\right)\right)\left(h_{(4)} \cdot g\right) \otimes h_{(2)} \cdot f_{(0)} \\
& =\sum \rho\left(h_{(1)}\right) \rho\left(S\left(f_{(1)}\right)\right) \rho\left(S\left(h_{(3)}\right)\right) \rho\left(h_{(4)}\right) g \rho\left(S\left(h_{(5)}\right)\right) \otimes h_{(2)} \cdot f_{(0)} \\
& =\sum \rho\left(h_{(1)}\right) \rho\left(S\left(f_{(1)}\right)\right) g \rho\left(S\left(h_{(4)}\right)\right) \otimes \rho\left(h_{(2)}\right) f_{(0)} \rho\left(S\left(h_{(3)}\right)\right) \\
& =\sum \rho\left(h_{(1)}\right)\left(\eta(f \# g) \rho\left(S\left(h_{(2)}\right)\right)\right. \\
& =h \cdot \eta(f \# g)
\end{aligned}
$$

Now the adjoint action of $H$ on $\operatorname{End}_{k}(N \otimes M)$ is strongly inner. It follows that the $H$-action on $\operatorname{End}_{k}(M) \# \operatorname{End}_{k}(N)$ is strongly inner too. Thus the algebra isomorphism $\phi$ induces a strongly inner action of $H$ on $\operatorname{End}_{k}(M \otimes N)$. By Lemma 4.3 there is an $H$-module structure on $M \otimes N$ such that $M \otimes N$ is a new $H$-bimodule, and its induced $H$-action and coaction on $\operatorname{End}_{k}(M \otimes N)$ force $\operatorname{End}_{k}(M \otimes N)$ to be a Yetter-Drinfel'd $H$-module algebra. Therefore, $\phi$ is a Yetter-Drinfel'd $H$-module algebra isomorphism.

Recall from [8] that for a faithfully projective $H$-bimodule $M$ the $k$-dual $M^{*}$ admits two types of $H$-bimodule structures:

Type 1: $(h \cdot p)(m)=\sum p(S(h) \cdot m)$, and $\chi(p)(m)=\sum p\left(m_{(0)}\right) \otimes S^{-1}\left(m_{(1)}\right)$.

Type 2: $(h \cdot p)(m)=\sum p\left(S^{-1}(h) \cdot m\right)$, and $\chi(p)(m)=\sum p\left(m_{(0)}\right) \otimes S\left(m_{(1)}\right)$.

Here $h \in H, p \in M^{*}$ and $m \in M$. The $H$-bimodule structures of type 1 induce the $H$-structures of $\operatorname{End}(M)$, and those of type 2 induce the $H$-structures of $\operatorname{End}(M)^{o p}$.

Lemma 4.4. Suppose that $M$ is a faithfully projective $H$-bimodule. Then

$$
\iota: \operatorname{End}(M)^{o p} \cong \operatorname{End}\left(M^{*}\right), f^{o p} \mapsto f^{*}
$$

is an $H$-bimodule algebras isomorphism.

Proof. The proof is the same as the one of $[8,(4.7)]$. Note that here $M^{*}$ as an $H$ bimodule admits the $H$-structures of type 2, and $\operatorname{End}_{k}(M)^{o p}$ has the $H$-structures given by (6) and (7). 
We have to show that $A_{\alpha}=\operatorname{End}\left(H_{\alpha}\right)$ is an $H$-Azumaya algebra. First let us define a new $H$-bimodule structure on $H$, denoted by $H_{\alpha}^{\prime}$, as follows:

$$
\begin{aligned}
h \cdot x & =\sum \alpha\left(h_{(2)}\right) x S^{-1}\left(h_{(1)}\right) \\
\chi^{\prime}(x) & =\sum x_{(1)} \otimes \alpha^{-1}\left(x_{(2)}\right)
\end{aligned}
$$

for $h \in H, x \in H_{\alpha}^{\prime}$. The $H$-bimodule $H_{\alpha}^{\prime}$ satisfies the compatibility condition

$$
\chi^{\prime}(h \cdot x)=\sum h_{(2)} \cdot x_{(0)} \otimes h_{(3)} x_{(1)} S^{-1}\left(\alpha^{-1}\left(h_{(1)}\right)\right)
$$

Lemma 4.5. $\operatorname{End}_{k}\left(H_{\alpha}^{\prime}\right)^{o p}$ is a Yetter-Drinfel'd H-module algebra, and

$$
\tau: \overline{\operatorname{End}_{k}\left(H_{\alpha}\right)} \longrightarrow \operatorname{End}_{k}\left(H_{\alpha}^{\prime}\right)^{o p}
$$

given by

$$
\tau(f)(m)=\sum f_{(0)}\left(f_{(1)} \cdot m\right)
$$

for $f \in \operatorname{End}_{k}\left(H_{\alpha}\right), m \in H_{\alpha}^{\prime}$, is an isomorphism of Yetter-Drinfel'd H-module algebras.

Proof. Using the notation $\rho$ we have that $\tau(f)=\sum \rho\left(f_{(1)}\right) \circ f_{(0)}=\sum f_{(0)} \rho\left(f_{(1)}\right)$ is well-defined. $\tau$ is an algebra map since

$$
\begin{aligned}
\tau(\bar{f} \circ \bar{g}) & =\sum \tau\left(\overline{g_{(0)}\left(g_{(1)} \cdot f\right)}\right) \\
& =\sum g_{(0)}\left(g_{(2)} \cdot f\right)_{(0)} \rho\left(\left(g_{(2)} \cdot f\right)_{(1)} g_{(1)}\right) \\
& =\sum g_{(0)}\left(g_{(3)} \cdot f_{(0)}\right) \rho\left(g_{(4)} f_{(1)} S^{-1}\left(g_{(2)}\right) g_{(1)}\right) \\
& =\sum g_{(0)}\left(g_{(1)} \cdot f_{(0)}\right) \rho\left(g_{(2)} f_{(1)}\right) \\
& =\sum g_{(0)} \rho\left(g_{(1)}\right) f_{(0)} \rho\left(S\left(g_{(2)}\right)\right) \rho\left(g_{(3)} f_{(1)}\right) \\
& =\sum g_{(0)} \rho\left(g_{(1)}\right) f_{(0)} \rho\left(f_{(1)}\right) \\
& =\sum \tau(\bar{g}) \tau(\bar{f})
\end{aligned}
$$

Also, $\tau$ is $H$-linear:

$$
\begin{aligned}
\tau(h \cdot \bar{f}) & =\sum\left(h_{(2)} \cdot f_{(0)}\right) \rho\left(h_{(3)} f_{(1)} S^{-1}\left(h_{(1)}\right)\right) \\
& =\sum \rho\left(h_{(2)}\right) f_{(0)} \rho\left(S\left(h_{(3)}\right) h_{(4)} f_{(1)} S^{-1}\left(h_{(1)}\right)\right) \\
& =\sum \rho\left(h_{(2)}\right) f_{(0)} \rho\left(f_{(1)}\right) \rho\left(S^{-1}\left(h_{(1)}\right)\right) \\
& =\sum h \cdot \tau(\bar{f})
\end{aligned}
$$

$\tau$ is $H$-colinear: for $m \in H$, keep the comodule structures $\chi^{\prime}(x)=\sum x_{(1)} \otimes$ $\alpha^{-1}\left(x_{(2)}\right) \in H_{\alpha}^{\prime} \otimes H$ and $\chi(x)=\Delta(x)=\sum x_{(1)} \otimes x_{(2)} \in H_{\alpha} \otimes H$, in mind. 
Then for $m \in H, \bar{f} \in \overline{\operatorname{End}\left(H_{\alpha}\right)}$, we have

$$
\begin{aligned}
& \chi(\tau(\bar{f}))(m)= \sum\left[\tau(\bar{f})\left(m_{(0)}\right)\right]_{(0)} \otimes\left[\tau(\bar{f})\left(m_{(0)}\right)\right]_{(1)} S\left(m_{(1)}\right) \\
&= \sum\left[\tau(\bar{f})\left(m_{(1)}\right)\right]_{(1)} \otimes \alpha^{-1}\left(\left[\tau(\bar{f})\left(m_{(1)}\right)\right]_{(2)}\right) S\left(\alpha^{-1}\left(m_{(2)}\right)\right) \\
&= \sum\left[f_{(0)}\left(f_{(1)} \cdot m_{(1)}\right)\right]_{(1)} \otimes \alpha^{-1}\left(\left[f_{(0)}\left(f_{(1)} \cdot m_{(1)}\right)\right]_{(2)} S\left(m_{(2)}\right)\right) \\
&= \sum\left[f_{(0)}\left(\left(f_{(1)} \cdot m_{(1)}\right)_{(1)}\right)\right]_{(1)} \\
& \otimes \alpha^{-1}\left(\left(f_{(1)} \cdot m_{(1)}\right)_{(3)} S^{-1}\left(\left(f_{(1)} \cdot m_{(1)}\right)_{(2)}\right)\right. \\
&\left.\quad \times\left[f_{(0)}\left(\left(f_{(1)} \cdot m_{(1)}\right)_{(1)}\right)\right]_{(2)} S\left(m_{(2)}\right)\right) \\
&= \sum f_{(0)}\left(\left(f_{(2)} \cdot m_{(1)}\right)_{(1)}\right) \otimes \alpha^{-1}\left(\left(f_{(2)} \cdot m_{(1)}\right)_{(2)} f_{(1)} S\left(m_{(2)}\right)\right) \\
&= \sum f_{(0)}\left(f_{(3)} \cdot m_{(1)}\right) \otimes \alpha^{-1}\left(\alpha\left(f_{(4)}\right) m_{(2)} S^{-1}\left(f_{(2)}\right) f_{(1)} S\left(m_{(3)}\right)\right) \\
&= \sum f_{(0)}\left(f_{(1)} \cdot m\right) \otimes f_{(2)} \\
&= \sum \tau\left(\overline{f_{(0)}}\right)(m) \otimes f_{(1)}
\end{aligned}
$$

This implies that $\chi(\tau(\bar{f}))=\sum \tau\left(\overline{f_{(0)}}\right) \otimes f_{(1)}$. Finally, it is easy to see from the $H$-colinearity of $\tau$ that $\tau$ has an inverse $\tau^{-1}$ defined as follows:

$$
\tau^{-1}(g)(m)=\overline{\sum g_{(0)} \rho\left(S\left(g_{(1)}\right)\right)}
$$

for $g \in \operatorname{End}_{k}\left(H_{\alpha}^{\prime}\right)^{o p}, m \in H_{\alpha}$.

Lemma 4.6. The algebra $A_{\alpha}$ defined above is an $H$-Azumaya algebra.

Proof. By (4.2), (4.4) and (4.5), $A_{\alpha} \# \overline{A_{\alpha}} \cong \operatorname{End}\left(H_{\alpha} \otimes H_{\alpha}^{\prime *}\right)$ as Yetter-Drinfel'd $H$-module algebras. It follows that $A_{\alpha} \# \overline{A_{\alpha}}$ is an Azumaya algebra. The canonical map

$$
F: A_{\alpha} \# \overline{A_{\alpha}} \longrightarrow \operatorname{End}\left(A_{\alpha}\right) ; F(a \# \bar{b})(c)=\sum a c_{(0)}\left(c_{(1)} \cdot b\right)
$$

is a Yetter-Drinfel'd $H$-module algebra map. It is readily seen that $F$ restricted to the center $k$ is the identity. It follows that $F$ is a monomorphism. Now $F$ is an isomorphism, since both sides have the same rank and are common Azumaya algebras. Similarly, the other canonical map $G$ is an isomorphism too. Therefore, $A_{\alpha}$ is an $H$-Azumaya algebra.

Let us call a Yetter-Drinfel'd $H$-module algebra which is the endomorphism ring of a faithfully projective $H$-bimodule a quasi-elementary Yetter-Drinfel'd $H$ module algebra. Following the notations introduced in [11], denote by $\mathrm{BT}(k, H)$ the subset of $\mathrm{BQ}(k, H)$ consisting of classes that are represented by quasi-elementary $H$-Azumaya algebras. $\mathrm{BT}(k, H)$ is closed under the multiplication \# in $\mathrm{BQ}(k, H)$, but we do not know if it is a subgroup of $\mathrm{BQ}(k, H)$. If Lemma (4.5) holds for any quasi-elementary $H$-Azumaya algebras, then $\mathrm{BT}(k, H)$ is a subgroup. This holds for example if $H$ is commutative, cocommutative and faithfully projective, cf. $[6,11]$. In general, we have a group anti-homomorphism

$$
\pi: \operatorname{Aut}_{\text {Hopf }}(H) \longrightarrow \mathrm{BT}(k, H), \alpha \mapsto\left[A_{\alpha}\right]
$$

Proposition 4.7. $\pi$ is an anti-homomorphism. 
Proof. Let $\alpha, \beta \in \operatorname{Aut}_{\text {Hopf }}(H)$, and consider the $H$-bimodules $H_{\alpha}, H_{\beta}$. Define an $H$-bimodule $M=H_{\alpha} \otimes H_{\beta}$ with $H$-structures given by

$$
h \cdot(x \otimes y)=\sum h_{(1)} \cdot x \otimes \alpha\left(h_{(2)}\right) \cdot y
$$

and

$$
\chi(x \otimes y)=\sum x_{(1)} \otimes y_{(1)} \otimes y_{(2)} x_{(2)}
$$

Then $\operatorname{End}_{k}(M)$ with the induced $H$-structures from $M$ is a Yetter-Drinfel'd $H$ module algebra. One may check that (26) is indeed induced by $\eta$ and $\phi$ described in (4.2). For $h \in H, m=x \otimes y \in M, M$ satifies the compatibility condition

$$
\chi(h \cdot m)=h_{(2)} \cdot m_{(0)} \otimes \beta \alpha\left(h_{(3)}\right) m_{(1)} S^{-1}\left(h_{(1)}\right)
$$

Take $\beta=\alpha^{-1}$. Then $M=H_{\alpha} \otimes H_{\alpha^{-1}}$ is a faithfully projective Yetter-Drinfel'd $H$-module. It follows that $\left[A_{\alpha} \# A_{\alpha^{-1}}\right]=\left[\operatorname{End}_{k}(M)\right]=1$ in $\mathrm{BQ}(k, H)$, and hence $\left[A_{\alpha}\right]^{-1}=\left[A_{\alpha^{-1}}\right]$ in $\mathrm{BQ}(k, H)$.

Next let us look at the $H$-bimodule $N=M \otimes H_{\gamma}$, where $\gamma=(\beta \alpha)^{-1}$. The comodule structure of $N$ is the usual one, but the $H$-module structure is given by

$$
h \cdot(m \otimes x)=\sum h_{(1)} \cdot m \otimes \beta \alpha\left(h_{(2)}\right) \cdot x
$$

As shown in (4.2), $\phi: \operatorname{End}(M) \# A_{\gamma} \cong \operatorname{End}(N)$ as Yetter-Drinfel'd $H$-module algebras. However, $N$ is a faithfully projective Yetter-Drinfel'd $H$-module. In fact, for $h \in H, n=m \otimes x \in N$,

$$
\begin{aligned}
& \chi(h \cdot n)=\sum \chi\left(h_{(1)} \cdot m \otimes \beta \alpha\left(h_{(2)}\right) \cdot x\right) \\
&= \sum h_{(2)} \cdot m_{(0)} \otimes \beta \alpha\left(h_{(5)}\right) \cdot x_{(0)} \\
& \otimes \gamma \beta \alpha\left(h_{(6)}\right) x_{(1)} S^{-1}\left(\beta \alpha\left(h_{(4)}\right)\right) \beta \alpha\left(h_{(3)}\right) m_{(1)} S^{-1}\left(h_{(1)}\right) \\
&= \sum h_{(2)} \cdot\left(m_{(0)} \otimes x_{(0)}\right) \otimes h_{(3)} x_{(1)} m_{(1)} S^{-1}\left(h_{(1)}\right)
\end{aligned}
$$

This fact entails that $\left[\operatorname{End}_{k}(M)\right]=\left[A_{\gamma}\right]^{-1}$. Therefore, $\left[A_{\alpha}\right]\left[A_{\beta}\right]=\left[A_{\alpha} \# A_{\beta}\right]=$ $\left[\operatorname{End}_{k}(M)\right]=\left[A_{\gamma}\right]^{-1}=\left[A_{\gamma^{-1}}\right]=\left[A_{\beta \alpha}\right]$. This finishes the proof.

To a Hopf algebra automorphism $\alpha$ of $H$, we may associate an element $\theta(\alpha)$ of Aut $(\mathrm{E}(H))$ given by

$$
\Theta(\alpha)(g \bowtie \lambda)=\alpha^{-1}(g) \bowtie \alpha^{*}(\lambda)
$$

This is well-defined since $\alpha^{-1}(g) \bowtie \alpha^{*}(\lambda)$ is in $\mathrm{E}(H)$ for any $g \bowtie \lambda \in \mathrm{E}(H)$. It is clear that $\Theta\left(\alpha^{-1}\right)$ is an inverse for $\Theta(\alpha)$ in $\operatorname{Aut}(\mathrm{E}(H))$. Thus we have obtained an anti-homomorphism $\Theta: \operatorname{Aut}_{\text {Hopf }}(H) \longrightarrow \operatorname{Aut}(\mathrm{E}(H))$. We now have to clarify the relationship between $\Theta$ and the anti-homomorphism $\pi$.

Theorem 4.8. With notations as above, the following diagram is commutative:

$$
\begin{array}{ccc}
\operatorname{Aut}_{\text {Hopf }}(H) & \stackrel{\Theta}{\longrightarrow} & \operatorname{Aut}(\mathrm{E}(H)) \\
\downarrow \pi & & \uparrow \beta \\
\mathrm{BT}(k, H) & \hookrightarrow & \mathrm{BQ}(k, H) .
\end{array}
$$


Proof. Given $\alpha \in \operatorname{Aut}_{\text {Hopf }}(H), A_{\alpha}$ is an $H$-Azumaya algebra. To each element $\sigma=g \bowtie \lambda \in \mathrm{E}(H)$ we associate the element $\psi_{\sigma} \in A_{\alpha}$ as follows: for $x \in H_{\alpha}$,

$$
\psi_{\sigma}(x)=\sum \alpha^{-1}(g) \cdot x_{(0)} \lambda\left(x_{(1)}\right)
$$

For $h \in H, x \in H_{\alpha}$, we have

$$
\begin{aligned}
\psi_{\sigma}(h \cdot x) & =\sum \alpha^{-1}(g) h_{(2)} \cdot x_{(0)} \lambda\left(\alpha\left(h_{(3)}\right) x_{(1)} S^{-1}\left(h_{(1)}\right)\right) \\
& =\sum \alpha^{-1}(g) h_{(2)} \cdot x_{(0)} \alpha^{*}(\lambda)\left(h_{(3)}\right) \lambda\left(x_{(1)}\right) \lambda^{-1}\left(h_{(1)}\right) \\
& =\sum \alpha^{-1}(g)\left(\alpha^{*}(\lambda)-h_{(2)}\right) \cdot x_{(0)} \lambda\left(x_{(1)}\right) \lambda^{-1}\left(h_{(1)}\right) \\
& =\sum\left(h_{(2)}-\alpha^{*}(\lambda)\right) \alpha^{-1}(g) \cdot x_{(0)} \lambda\left(x_{(1)}\right) \lambda^{-1}\left(h_{(1)}\right) \\
& =\sum\left(h-\lambda^{-1} \alpha^{*}(\lambda)\right) \psi_{\sigma}(x)
\end{aligned}
$$

The above equality yields the following:

$$
\begin{aligned}
\left(h \cdot \psi_{\sigma}\right)(x) & =\sum h_{(1)} \psi_{\sigma}\left(S\left(h_{(2)}\right) \cdot x\right) \\
& =\sum h_{(1)}\left(S\left(h_{(2)}\right)-\lambda^{-1} \alpha^{*}(\lambda)\right) \psi_{\sigma}(x) \\
& =\sum h_{(1)} S\left(h_{(2)}\right)\left\langle S\left(h_{(3)}\right), \lambda^{-1} \alpha^{*}(\lambda)\right\rangle \psi_{\sigma}(x) \\
& =\sum\left\langle h, \alpha^{*}\left(\lambda^{-1}\right) \lambda\right\rangle \psi_{\sigma}(x)
\end{aligned}
$$

It follows that $h \cdot \psi_{\sigma}=\left\langle h, \alpha^{*}\left(\lambda^{-1}\right) \lambda\right\rangle \psi_{\sigma}$ for any $h \in H$. For the $H$-coaction on $\psi_{\sigma}$, we have

$$
\begin{aligned}
\chi\left(\psi_{\sigma}(x)\right) & =\sum \alpha^{-1}(g) \cdot x_{(0)} \otimes g x_{(1)} S^{-1}\left(\alpha^{-1}(g)\right) \lambda\left(x_{(2)}\right) \\
& =\sum \alpha^{-1}(g) \cdot x_{(0)} \otimes \lambda\left(x_{(1)}\right) x_{(2)} g \alpha^{-1}\left(g^{-1}\right) \\
& =\sum \psi_{\sigma}\left(x_{(0)}\right) \otimes x_{(1)} g \alpha^{-1}\left(g^{-1}\right)
\end{aligned}
$$

An easy computation yields $\chi\left(\psi_{\sigma}\right)=\psi_{\sigma} \otimes g \alpha^{-1}\left(g^{-1}\right)$.

It remains to be proved that $\psi_{\sigma} \in \Phi(\rho(\sigma))$. For $f \in A_{\alpha}, \sigma(f) \equiv \rho(\sigma)(f)=$ $\sum g \cdot f_{(0)} \lambda\left(f_{(1)}\right)$. Then for $x \in H_{\alpha}$, we have

$$
\begin{aligned}
\sigma(f) \psi_{\sigma}(x) & =\sum \sigma(f)\left(\alpha^{-1}(g) \cdot x_{(0)}\right) \lambda\left(f_{(1)}\right) \\
& =\sum\left(g \cdot f_{(0)}\right)\left(\alpha^{-1}(g) \cdot x_{(0)}\right) \lambda\left(x_{(1)} f_{(1)}\right) \\
& =\sum g \cdot f_{(0)}\left(g^{-1} \alpha^{-1}(g) \cdot x_{(0)}\right) \lambda\left(x_{(1)} f_{(1)}\right)
\end{aligned}
$$

Now set $\bar{g}=g \alpha^{-1}\left(g^{-1}\right)$; then

$$
\begin{aligned}
\sum & \psi_{\sigma(0)}\left(\psi_{\sigma(1)} \cdot f\right)(x)=\psi_{\sigma}(\bar{g} \cdot f)(x) \\
& =\psi_{\sigma}\left(\bar{g} \cdot f\left(\bar{g}^{-1} \cdot x\right)\right) \\
& =\sum \alpha^{-1}(g) \bar{g} \cdot f\left(\bar{g}^{-1} \cdot x\right)_{(0)} \lambda\left(\alpha(\bar{g}) f\left(\bar{g}^{-1} \cdot x\right)_{(1)} S^{-1}(\bar{g})\right) \\
& =\sum g \cdot f\left(\left(\bar{g}^{-1} \cdot x_{(0)}\right)_{(0)}\right)_{(0)} \lambda\left(x_{(1)} S^{-1}\left(\left(\bar{g}^{-1} \cdot x_{(0)}\right)_{(1)}\right) f\left(\left(\bar{g}^{-1} \cdot x_{(0)}\right)_{(0)}\right)_{(1)}\right) \\
& =\sum g \cdot f_{(0)}\left(\bar{g}^{-1} \cdot x_{(0)}\right) \lambda\left(x_{(1)} f_{(1)}\right)
\end{aligned}
$$


Therefore, $\sigma(f) \psi_{\sigma}=\sum \psi_{\sigma(0)}\left(\psi_{\sigma(1)} \cdot f\right)$, and we have proved that $\psi_{\sigma} \in \Phi(\rho(\sigma))$. By the definition of the homomorphism $\alpha_{A_{\alpha}}$, we know that $\alpha_{A_{\alpha}}(\sigma)=g \alpha^{-1}\left(g^{-1}\right) \bowtie$ $\alpha^{*}\left(\lambda^{-1}\right) \lambda$, and it follows that

$$
\beta_{A_{\alpha}}(\sigma)=\sigma \alpha_{A_{\alpha}}(\sigma)^{-1}=\alpha^{-1}(g) \bowtie \alpha^{*}(\lambda)=\Theta(\alpha)(\sigma)
$$

This finishes our proof.

The above commutative diagram yields $\operatorname{Ker}(\pi) \subseteq \operatorname{Ker}(\Theta)$. If $H$ is commutative, cocommutative and faithfully projective, then $\Theta$ is injective, and hence $\operatorname{Aut}_{\text {Hopf }}(H)$ is anti-isomorphic to some subgroup of $\mathrm{BQ}(k, H)$; cf. [6, 11].

In the rest of this section, we consider the action of $\mathrm{Aut}_{\mathrm{Hopf}}$ on $\mathrm{BQ}(k, H)$. We will show that, in some sense, this action can be measured by the map $\beta$.

Let $A$ be an $H$-Azumaya algebra, and $\alpha$ a Hopf algebra automorphism of $H$. Consider the Yetter-Drinfel'd $H$-module algebra $A(\alpha)$, which equals $A$ as a $k$-algebra, but with $H$-structure $\left(A(\alpha), \neg, \chi^{\prime}\right)$ given by

$$
h \rightarrow a=\alpha(h) \cdot a \text { and } \chi^{\prime}(a)=\sum a_{(0)} \otimes \alpha^{-1}\left(a_{(1)}\right)=\left(1 \otimes \alpha^{-1}\right) \chi(a)
$$

for all $a \in A(\alpha), h \in H$.

Lemma 4.9. Let $A, B$ be $H$-Azumaya algebras. If $\alpha$ is a Hopf algebra automorphism of $H$, then $A(\alpha)$ is an $H$-Azumaya algebra, and $(A \# B)(\alpha) \cong A(\alpha) \# B(\alpha)$.

Proof. The second part is clear. We show the first part. Let $e=\sum x_{i} \# \overline{y_{i}}$ be the $H$-separability idempotent of $A \# \bar{A}$. It is readily verified that $e$ is still the $H$-separability idempotent of $A(\alpha) \# \overline{A(\alpha)}$, by (2.14). So $A(\alpha)$ is $H$-separable. Now

$$
A(\alpha)^{A(\alpha)}=\left\{x \in A(\alpha) \mid a x=\sum x_{(0)}\left(\alpha^{-1}\left(x_{(1)}\right) \rightarrow a\right), \forall a \in A(\alpha)\right\}=A^{A}
$$

implies that $A(\alpha)$ is right central. Similarly $A_{\alpha}$ is left central. Therefore $A(\alpha)$ is $H$-Azumaya.

(4.9) allows us to define the group action of $\operatorname{Aut}_{\text {Hopf }}(H)$ on $\mathrm{BQ}(k, H)$ through $\alpha([A])=[A(\alpha)]$ for $\alpha \in \operatorname{Aut}_{\text {Hopf }}(H)$ and $[A] \in \mathrm{BQ}(k, H)$. On the other hand, there exists a natural action of $\operatorname{Aut}_{\text {Hopf }}(H)$ on $\operatorname{Aut}(\mathrm{E}(H))$ by inner automorphisms, i.e.,

$$
\alpha(f)=\Theta(\alpha) f \Theta\left(\alpha^{-1}\right), \text { for } f \in \operatorname{Aut}(\mathrm{E}(H))
$$

Proposition 4.10. The map $\beta$ respects the $\operatorname{Aut}_{\mathrm{Hopf}}(H)$-action, that is, $\beta_{A(\alpha)}=$ $\Theta(\alpha) \beta_{A} \Theta\left(\alpha^{-1}\right)$.

Proof. For $\sigma=g \bowtie \lambda \in \mathrm{E}(H)$, set $\alpha_{A}(\sigma)=p \bowtie \eta$. Choose an element $u \in$ $\Phi_{A}(\rho(\sigma))$; then

for $h \in H$.

$$
\chi(u)=u \otimes p \text { and } h \cdot u=\eta(h) u
$$

Let us look at the element $u \in A(\alpha)$. By the definition of $A(\alpha)$ we know that $\chi^{\prime}(u)=u \otimes \alpha^{-1}(p)$, and $h \rightarrow u=\alpha^{*}(\eta)(h) u$. In fact, $u \in \Phi_{A(\alpha)}(\rho(\Theta(\alpha)(\sigma)))$ since

$$
\begin{aligned}
u\left(\alpha^{-1}(g) \rightarrow a\right) & =u(g \cdot a) \\
& =\sum g \cdot a_{(0)} \lambda\left(a_{(1)}\right) u \\
& =\sum \alpha^{-1}(g) \rightarrow a_{(0)} \alpha^{*}(\lambda)\left(\alpha^{-1}\left(a_{(1)}\right)\right) u \\
& =\Theta(\alpha)(\sigma)(a) u
\end{aligned}
$$


for any $a \in A(\alpha)$. This implies that $\Phi_{A(\alpha)}(\rho(\Theta(\alpha)(\sigma)))=\Theta(\alpha)\left(\alpha_{A}(\sigma)\right)$, and hence we have $\Phi_{A(\alpha)}(\rho(\sigma))=\Theta(\alpha) \alpha_{A} \Theta\left(\alpha^{-1}\right)(\sigma)$. Now

$$
\begin{aligned}
\beta_{A(\alpha)}(\sigma) & =\sigma \alpha_{A(\alpha)}(\sigma) \\
& =\sigma\left(\Theta(\alpha) \alpha_{A} \Theta\left(\alpha^{-1}\right)(\sigma)\right)^{-1} \\
& =\Theta(\alpha)\left(\Theta\left(\alpha^{-1}\right)(\sigma)\left(\alpha_{A} \Theta\left(\alpha^{-1}\right)(\sigma)\right)^{-1}\right) \\
& =\Theta(\alpha) \beta_{A} \Theta\left(\alpha^{-1}\right)(\sigma)
\end{aligned}
$$

Therefore, $\beta_{A(\alpha)}=\Theta(\alpha) \beta_{A} \Theta\left(\alpha^{-1}\right)$, and the map $\beta$ preserves the $\operatorname{Aut}_{\text {Hopf }}(H)$ action.

The above proposition suggests that the action of $\operatorname{Aut}_{\text {Hopf }}(H)$ on $\mathrm{BQ}(k, H)$ might be inner. Indeed, we have

Theorem 4.11. $\operatorname{Aut}_{\mathrm{Hopf}}(H)$ acts innerly on $\mathrm{BQ}(k, H)$. More precisely, for any $H$-Azumaya algebra $B$ and $\alpha \in \operatorname{Aut}_{\mathrm{Hopf}}(H)$, we have $[B(\alpha)]=\left[A_{\alpha}\right][B]\left[A_{\alpha^{-1}}\right]$.

Proof. It suffices to show that $B \# A_{\alpha} \cong A_{\alpha} \# B\left(\alpha^{-1}\right)$ for any $\alpha \in \operatorname{Aut}_{\text {Hopf }}(H)$. Identify $A_{\alpha}$ with $H_{\alpha} \otimes H_{\alpha}^{*}$ as described in the beginning of this section. The multiplication on $H_{\alpha} \otimes H_{\alpha}^{*}$ is the obvious one, that is,

$$
\left(m \otimes m^{*}\right)\left(n \otimes n^{*}\right)=m \otimes n^{*}\left\langle m^{*}, n\right\rangle .
$$

Recall that $H_{\alpha}$ satisfies the compatibility condition:

$$
\chi(h \cdot m)=\sum h_{(2)} \cdot m_{(0)} \otimes \alpha\left(h_{(3)}\right) m_{(1)} S^{-1}\left(h_{(1)}\right) .
$$

This implies that the $H$-bimodule $H_{\alpha}^{*}$ satisfies the compatibility condition

$$
\chi(h \cdot p)=\sum h_{(2)} \cdot p_{(0)} \otimes h_{(3)} p_{(1)} S^{-1}\left(\alpha\left(h_{(1)}\right)\right)
$$

Without ambiguity, we keep the notation $(\neg,(1 \otimes \alpha) \chi)$ for $B\left(\alpha^{-1}\right)$, and $(\cdot, \chi)$ for $B$. We define the map

$$
\phi: B \# A_{\alpha} \longrightarrow A_{\alpha} \# B\left(\alpha^{-1}\right)
$$

by

$$
\begin{aligned}
\phi\left(b \# m \otimes m^{*}\right) & =\sum S\left(b_{(1)}\right) \cdot m \otimes m_{(0)}^{*} \# m_{(1)}^{*} \rightarrow b_{(0)} \\
& =\sum S\left(b_{(1)}\right) \cdot m \otimes m_{(0)}^{*} \# \alpha^{-1}\left(m_{(1)}^{*}\right) \cdot b_{(0)}
\end{aligned}
$$

Let us show that $\phi$ is $H$-linear and $H$-colinear. The long, straightforward and tedious verification of the fact that $\phi$ is an algebra map is left to the reader. First, $\phi$ is $H$-linear since

$$
\begin{aligned}
\phi & \left(h_{(1)} \cdot b \# h_{(2)} \cdot m \otimes h_{(3)} \cdot m^{*}\right) \\
& =\sum S\left(h_{(3)} b_{(1)} S^{-1}\left(h_{(1)}\right)\right) h_{(4)} \cdot m \otimes\left(h_{(5)} \cdot m^{*}\right)_{(0)} \#\left(h_{(5)} \cdot m^{*}\right)_{(1)} \rightarrow\left(h_{(2)} \cdot b_{(0)}\right) \\
& =\sum h_{(1)} S\left(b_{(1)}\right) \cdot m \otimes h_{(4)} \cdot m_{(0)}^{*} \# \alpha^{-1}\left(h_{(5)} m_{(1)}^{*} S^{-1}\left(\alpha\left(h_{(3)}\right)\right)\right) h_{(2)} \cdot b_{(0)} \\
& =\sum h_{(1)} S\left(b_{(1)}\right) \cdot m \otimes h_{(2)} \cdot m_{(0)}^{*} \# \alpha^{-1}\left(h_{(3)}\right) \alpha^{-1}\left(m_{(1)}^{*}\right) \cdot b_{(0)} \\
& =h \cdot \phi\left(b \# m \otimes m^{*}\right)
\end{aligned}
$$


Next, $\phi$ is $H$-colinear:

$$
\begin{aligned}
& \chi\left(\phi\left(b \# m \otimes m^{*}\right)\right) \\
&= \sum\left(S\left(b_{(1)}\right) \cdot m\right)_{(0)} \otimes m_{(0)}^{*} \\
& \#\left(m_{(2)}^{*} \neg b_{(0)}\right)_{(0)} \otimes\left(m_{(2)}^{*} \cdot b_{(0)}\right)_{(1)} m_{(1)}^{*}\left(S\left(b_{(1)}\right) \cdot m\right)_{(1)} \\
&= \sum S\left(b_{(3)}\right) \cdot m_{(0)} \otimes m_{(0)}^{*} \\
& \# m_{(3)}^{*} \neg b_{(0)} \otimes m_{(4)}^{*} \alpha\left(b_{(1)}\right) S^{-1}\left(m_{(2)}^{*}\right) m_{(1)}^{*} \alpha\left(S\left(b_{(2)}\right)\right) m_{(1)} b_{(4)} \\
&= \sum S\left(b_{(1)}\right) \cdot m_{(0)} \otimes m_{(0)}^{*} \# m_{(1)}^{*} \rightarrow b_{(0)} \otimes m_{(2)}^{*} m_{(1)} b_{(2)} \\
&= \sum \phi\left(b_{(0)} \# m_{(0)} \otimes m_{(0)}^{*}\right) \otimes m_{(1)}^{*} m_{(1)} b_{(1)}
\end{aligned}
$$

Finally, $\phi$ is an isomorphism with inverse $\phi^{-1}$ defined by

$$
\phi^{-1}\left(m \otimes m^{*} \# b\right)=\sum\left(S\left(m_{(1)}^{*}\right) \rightarrow b_{(0)}\right)_{(0)} \#\left(S\left(m_{(1)}^{*}\right) \rightarrow b_{(0)}\right)_{(1)} \cdot m \otimes m_{(0)}^{*} .
$$

We leave the verification to the reader.

\section{REFERENCES}

[1] M. Beattie, Brauer groups of $H$-module and $H$-dimodule algebras, Queen's University, Kingston, Ontario, 1976.

[2] M. Beattie, A direct sum decomposition for the Brauer group of $H$-module algebras, $J$. Alg. 43 (1976), 686-693. MR 56:333

[3] M. Beattie, The Brauer group of central separable G-Azumaya algebras, J. Alg. 54 (1978), 516-525. MR 81h:16010

[4] S. Caenepeel, A note on inner actions of Hopf algebras, Proc. Amer. Math. Soc. 113 (1991), 31-39. MR 91k: 16026

[5] S. Caenepeel, Computing the Brauer-Long group of a Hopf algebra I: the cohomological theory, Israel J. Math. 72 (1990), 38-83. MR 92f:16046

[6] S. Caenepeel, Computing the Brauer-Long group of a Hopf algebra II: the Skolem-Noether theory, J. Pure Appl. Alg. 84 (1993),107-144. MR 93m:16026

[7] S. Caenepeel and M. Beattie, A cohomological approach to the Brauer-Long group and the groups of Galois extensions and strongly graded rings, Trans. Amer. Math. Soc. 324 (1991), 747-775. MR 91h:16030

[8] S. Caenepeel, F.Van Oystaeyen and Y.H.Zhang, Quantum Yang-Baxter module algebras, K-Theory 8 (1994), 231-255. MR 95e:16031

[9] L.N. Childs, G. Garfinkel and M. Orzech, The Brauer group of graded Azumaya algebras, Trans. Amer. Math. Soc. 175 (1973), 299-326. MR 50:2145

[10] L.N. Childs, The Brauer group of graded Azumaya algebras II: graded Galois extensions, Trans. Amer. Math. Soc. 204 (1975),137-160. MR 51:471

[11] A.P. Deegan, A subgroup of the generalized Brauer group of $\Gamma$-Azumaya algebras, J.London Math. Soc. 2 (1981), 223-240. MR 82j:16011

[12] V.G. Drinfel'd, Quantum groups, Proc. of the Int. Congress of Math., Berkeley, Ca. (1987), 798-819. MR 89f: 17017

[13] M.A. Knus and M. Ojanguren, Théorie de la descente et algèbres d'Azumaya, Lecture Notes in Math. 389, Springer-Verlag, Berlin, 1974. MR 54:5209

[14] F.W. Long, The Brauer group of dimodule algebras, J. Alg. 31 (1974), 559-601. MR 50:9941

[15] L.A. Lambe, D.E. Radford, Algebraic aspects of the quantum Yang-Baxter equation, J. Alg. 154 (1992), 228-288. MR 94b:17026

[16] S. Majid, Quasitriangular Hopf algebras and Yang-Baxter equations, Internat. J. Modern Phys. A(5) (1990), 1-91. MR 90k:16008

[17] S. Majid, Doubles of quasitriangular Hopf algebras, Comm Alg. 19 (1991), 3061-3073. MR 92k:16052

[18] S. Montgomery, Hopf algebras and their actions on rings, CBMS Regional Conf. Ser. Math., no. 82, Amer. Math. Soc., Providence, 1993. MR 94i:16019 
[19] M. Orzech, Brauer groups of graded algebras, Lecture Notes in Math. 549, Springer-Verlag, Berlin, 1976, 134-147. MR 56:8545

[20] M. Orzech, On the Brauer group of algebras having a grading and an action, Can. J. Math. 28 (1976), 533-552; correction, ibid. 32 (1980), 1523-1524. MR 53:8115; MR 82j:16014

[21] B. Pareigis, Non-additive ring and module theory IV, the Brauer group of a symmetric monoidal category, Lecture Notes in Math. 549, Springer-Verlag, Berlin, 1976, 112-133. MR 58:16834c

[22] D. E. Radford, Minimal quasitriangular Hopf algebras, J. Alg. 157 (1993), 285-315. MR 94c: 16052

[23] D.E. Radford and J. Towber, Yetter-Drinfel'd categories associated to an arbitrary bialgebra, J. Pure Appl. Algebra 87 (1993), 259-279. MR 94f:16060

[24] M.E. Sweedler, Hopf Algebras, Benjamin, 1969. MR 40:5705

[25] F. Van Oystaeyen and Y. H. Zhang, The Brauer group of a braided monoidal category, preprint 1996.

[26] C.T.C. Wall, Graded Brauer groups, J. Reine Angew. Math. 213 (1964), 187-199. MR 29:4771

[27] D.N. Yetter, Quantum groups and representations of monoidal categories, Math. Proc. Cambridge Philos. Soc. 108 (1990), 261-290. MR 91k:16028

Faculty of Applied Sciences, Free University of Brussels, Vub, Pleinlaan 2, B-1050 Brussels, Belgium

E-mail address: scaenepe@vnet3.vub.ac.be

Department of Mathematics, University of Antwerp, UiA, Universiteitsplein 1, B2610 WilRIJK, BELGiUM

E-mail address: francin@wins.uia.ac.be

E-mail address: zhang@wins.uia.ac.be 